\title{
Plasmonic Nanocrystal Arrays on Photonic Crystals with Tailored Optical Resonances
}

\author{
Juan Wang,* Hai Le-The, Theodosios Karamanos, Radius N.S. Suryadharma, Albert van den Berg, \\ Pepijn W. H. Pinkse, Carsten Rockstuhl, Lingling Shui,* Jan C. T. Eijkel, and Loes I. Segerink
}

Cite This: ACS Appl. Mater. Interfaces 2020, 12, 37657-37669

Read Online

ACCESS

Llll Metrics \& More

Article Recommendations

Supporting Information

ABSTRACT: Hierarchical plasmonic-photonic microspheres (PPMs) with high controllability in their structures and optical properties have been explored toward surface-enhanced Raman spectroscopy. The PPMs consist of gold nanocrystal (AuNC) arrays (3rd-tier) anchored on a hexagonal nanopattern (2nd-tier) assembled from silica nanoparticles $\left(\mathrm{SiO}_{2} \mathrm{NPs}\right)$ where the uniform microsphere backbone is termed the 1st-tier. The PPMs sustain both photonic stop band (PSB) properties, resulting from periodic $\mathrm{SiO}_{2} \mathrm{NP}$ arrangements of the 2nd-tier, and a surface plasmon resonance (SPR), resulting from AuNC arrays of the 3rd-tier. Thanks to the synergistic effects of the photonic crystal (PC) structure and the AuNC array, the electromagnetic (EM) field in

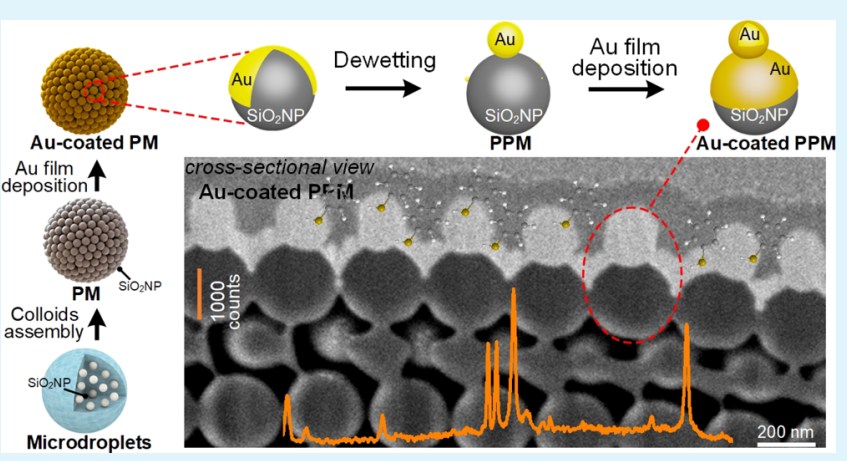
such a multiscale composite structure can tremendously be enhanced at certain wavelengths. These effects are demonstrated by experimentally evaluating the Raman enhancement of benzenethiol $(\mathrm{BT})$ as a probe molecule and are confirmed via numerical simulations. We achieve a maximum SERS enhancement factor of up to $\sim 10^{8}$ when the resonances are tailored to coincide with the excitation wavelength by suitable structural modifications. KEYWORDS: plasmonic-photonic microsphere, photonic stop band, slow light effect, localized surface plasmon resonance, surface-enhanced Raman spectroscopy

\section{INTRODUCTION}

Surface-enhanced Raman spectroscopy (SERS) has attracted increasing attention in both scientific research and practical applications due to its high sensitivity and specificity. It relies on enhancing the Raman signal from molecules close to a supporting SERS-active substrate, mostly made from noble metals. This method allows nondestructive and label-free detection of a wide range of molecules with near single molecule detection limit. ${ }^{1-3}$ Therefore, it has been applied in various fields such as chemistry, biology, medicine, and environmental science. ${ }^{4,5}$ The SERS phenomenon is mainly attributed to a combination of an electromagnetic (EM) enhancement (due to the excitation of surface plasmon polaritons, i.e., an excitation where the free electrons in the metal couple resonantly to a driving EM field) with a chemical enhancement (charge transfer (CT) between SERS-active substrates and analyte molecules). ${ }^{3,6,7}$ Generally, the contribution of the EM enhancement is much stronger than that of the CT enhancement. ${ }^{8}$ It has been reported that the EM enhancement can be manipulated by tailoring the characteristic dimensions and optical properties of the SERS-active substrates. ' A variety of SERS-active substrates/structures have been fabricated using top-down methods ${ }^{9}$ (e.g., electron-beam lithography and colloidal lithography), bottom-up methods ${ }^{10,11}$ (e.g., colloid assembly), or hybrid methods (top-down combined with bottom-up approach) $)^{11}$ to provide and control the localized surface plasmon resonance (LSPR) by varying the geometry of nanostructures (e.g., nanogap size and shape and periodicity), plasmonic resonance frequencies and modes, ${ }^{12}$ thereby resulting in an enhanced local EM field on demand. Nevertheless, the development of cost-effective, reproducible approaches to fabricate SERS-active substrates with high sensitivity and high enhancement factors (EFs) are still at stake and become an increasingly significant topic with the ultimate goal to eventually achieve near single molecule level detection. ${ }^{13}$

Noble metals have been mostly used as the base materials for SERS-active substrates (e.g., $\mathrm{Au}, \mathrm{Ag}$, and $\mathrm{Cu}$ ) as they possess unique surface plasmon resonance (SPR) properties upon illumination with incident light. ${ }^{14}$ Noble metal nanostructures can confine light into small volumes to enhance the local EM field close to their surface. Such greatly enhanced local EM field

Received: March 25, 2020

Accepted: July 28, 2020

Published: July 28, 2020 


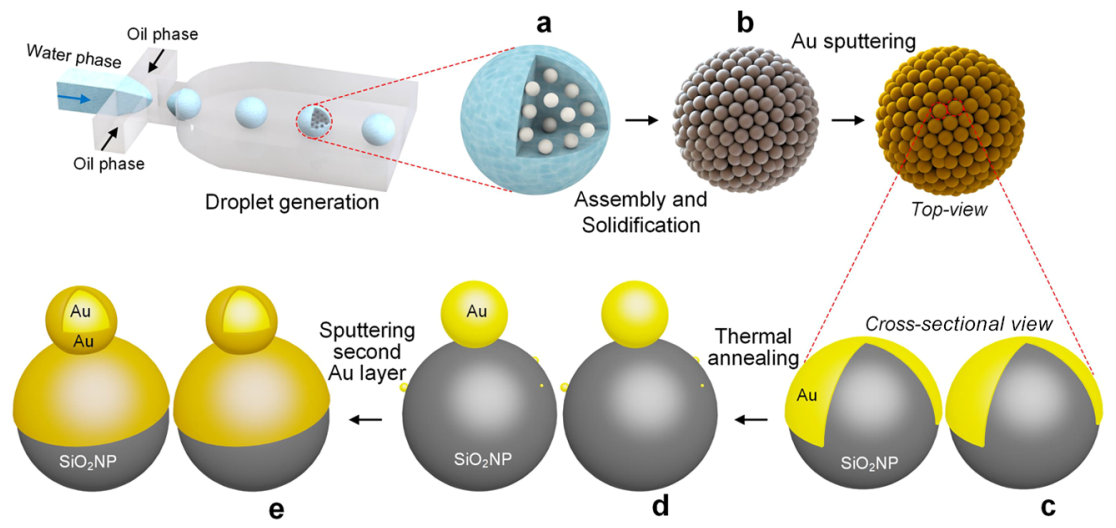

Figure 1. Schematic diagrams of the process to fabricate PPMs. (a) Microdroplets generated using a microfluidic droplet generator. (b) PM formed by $\mathrm{SiO}_{2} \mathrm{NP}$ assembly and evaporation-induced solidification. (c) Au-coated PM. (d) Formation of a PPM by thermal annealing the Au-coated PM. (e) A PPM coated with a second Au layer by sputtering. (Panels $(c-e)$ ) Close-up diagram of the corresponding single microsphere.

areas are defined as "hotspots" in SERS-active substrates, as in turn the weak Raman scattering signal is particularly enhanced if the molecules are located in these spatial locations. It has to be reminded that the EM SERS enhancement factor is approximately proportional to the fourth power of the electric field amplitude. $^{15}$ Many metal SERS-active substrates, such as colloidal metal nanoparticles (e.g., dimers and aggregates), metal nanopatterns, or three-dimensional (3D) plasmonic structures have been reported. ${ }^{16-18}$ However, either expensive equipment or complex chemical synthesis is required to fabricate these metal nanostructures.

It is worth noting that photonic crystal (PC) structures made from dielectrics have also long been considered for SERS application and not just metals. They are either used as SERSactive substrate themselves ${ }^{19}$ or serve as a substrate to provide structural support for metal films or metal nanoparticles. ${ }^{20} \mathrm{PC}$ structures consist of a periodic arrangement of materials with alternative permittivities. PC structures can tailor the propagation of light and can improve the light-matter interaction thanks to multiple scattering effects. Particularly, PC structures offer photonic stop bands (PSBs) in certain wavelength regions where the propagation of light along a specific direction is forbidden. Also, close to the edge of the PSB, the group velocity of light can be slowed down. ${ }^{21,22}$ For example, Qi et al. ${ }^{19}$ have reported a plasmon-free $\mathrm{TiO}_{2}$ photonic microarray as a SERS-active substrate. They explained the enhanced light - matter interaction by the repeated and multiple light scattering in their PC structures. It has been demonstrated that the SERS enhancement strongly depends on spectral alignment between the PSB and the laser wavelength used in the SERS measurements. ${ }^{19}$ PC structures with a PSB center near the laser excitation wavelength show lower SERS signal owing to the reduced local density of states. In contrast, PC structures with PSB edges in the proximity of the laser excitation wavelength show higher SERS signal that is explained by the increased local density of states that accompanies the slow light effect. ${ }^{19}$ In addition, our group has previously reported the fabrication of PC microspheres with metal-covered nanoarrays causing LSRP, which showed a high SERS EF $\left(>10^{7}\right)$, which we attributed to the high density "hotspots" confined in a single microsphere. ${ }^{11}$

However, herein, we mainly focus on the enhancement of the EM field by a composite structure, possessing both PSB and LSPR properties, to investigate the Raman enhancement performance. Till now, only a few studies ${ }^{23-25}$ have shown the fabrication of SERS-active substrates with both LSPR (resulting from the noble metal nanoparticles/2D nanoarrays/3D nanostructures) and PSB properties (resulting from the PC structures) to improve the enhanced EM field synergistically, thereby leading to a further enhanced SERS intensity. EM modeling of a bioinspired PC structure incorporated with $\mathrm{Au}$ nanocrystals for enhancing the localized electric field was explored by Zheng et al. ${ }^{26}$ They showed that the Auincorporated system can produce a stronger EM field due to the presence of the PC structure. Fränzl et al. ${ }^{25}$ have reported an SERS-active substrate by covering a one-dimensional PC with ordered metal nanoparticle arrays by electrochemical etching and the nanosphere lithography method, exhibiting a significant interaction of the plasmonic resonance with the PSB. Such an SERS-active substrate leads to a highly confined EM field at the interface between both structures and thus showing a significant enhancement in Raman signal with EFs up to $10^{5}$. Lee et al. ${ }^{27}$ have also demonstrated that the SERS behavior of amorphous $\mathrm{TiO}_{2}-\mathrm{Ag}$ nanoarchitectures depends on the plasmonicphotonic interference coupling where SERS-active units were fabricated by a two-step anodization on a titanium substrate and thermal annealing to obtain silver nanoparticles on top of the titanium dioxide nanotube (TNT) structures. However, there are still ample opportunities to improve the plasmonicphotonic structures for SERS enhancement in a facile and robust manner to render structures with more designability and controllability with respect to the hierarchical structures and the resulting optical properties of both LSPR and PSB.

In this work, a robust and cost-effective approach has been developed to fabricate hierarchical plasmonic-photonic microspheres (PPMs) by combining droplet microfluidics with metal film deposition and thermal annealing. The fabricated PPMs possess both PSB properties, resulting from periodic silica nanoparticle $\left(\mathrm{SiO}_{2} \mathrm{NP}\right)$ arrangements of the 2 nd-tier, and SPR properties, resulting from the AuNC arrays of the 3rd-tier. The uniform microsphere backbone is regarded as the 1st-tier. We investigated the SERS sensitivity dependent on the PSB edge of the PC structures by varying the size of the $\mathrm{SiO}_{2} \mathrm{NPs}$. We observe a complicated impact of the size of the $\mathrm{SiO}_{2} \mathrm{NP}$ on the achievable SERS enhancement because (i) the spectral position of the PSB is modified but also (ii) because the size of the $\mathrm{SiO}_{2} \mathrm{NP}$ affects the geometrical properties of the deposited metallic structures and with that the spectral position of the SPR. The complicated interplay can, nevertheless, be reproduced in dedicated full-wave optical simulations that we perform to provide further insights to the experimental results. We 

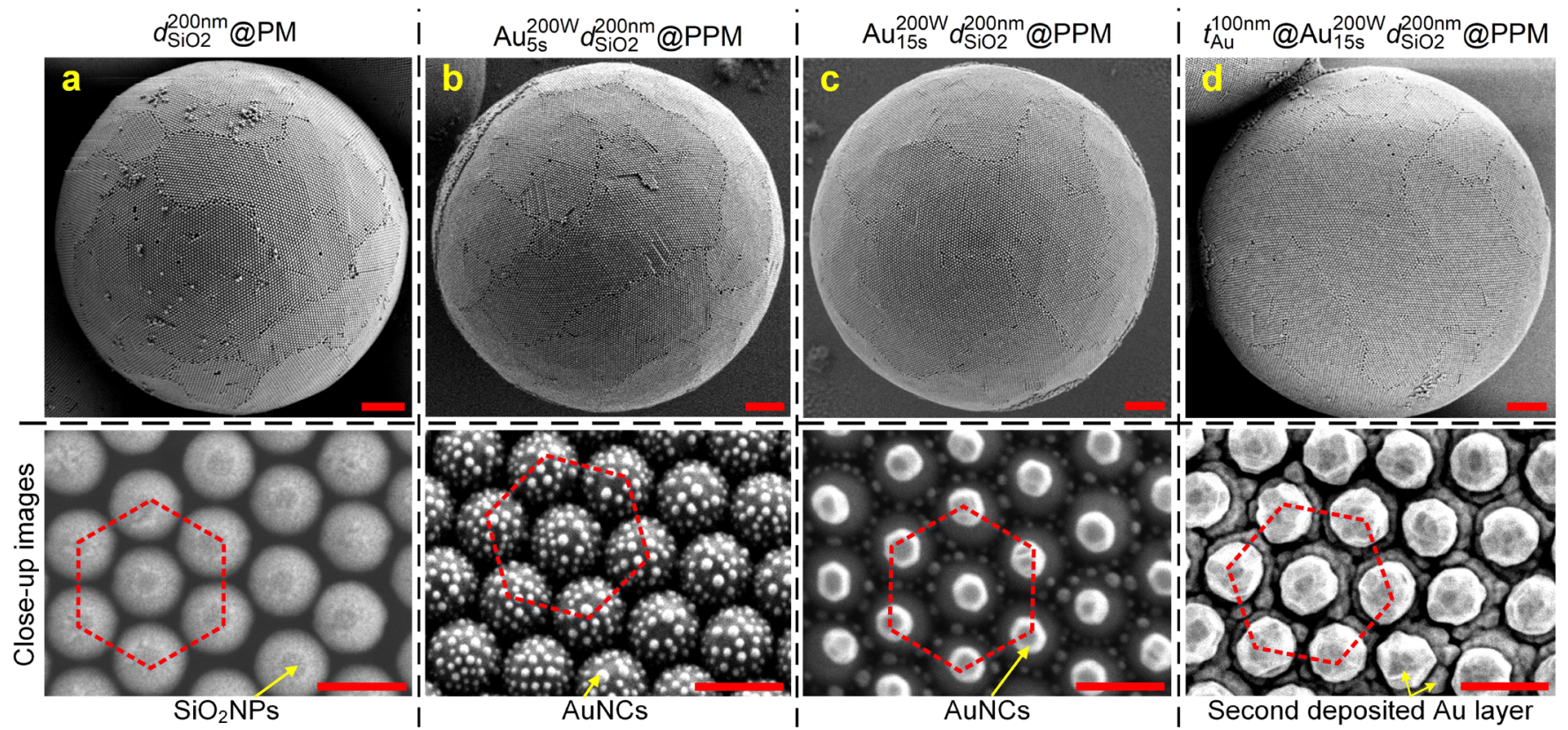

Figure 2. Top-view HR-SEM images (scale bar: $2 \mu \mathrm{m}$ ) of (a) a $d_{\mathrm{SiO} 2}^{200 \mathrm{~nm}} @ \mathrm{PM},(\mathrm{b})$ a $\mathrm{Au}_{5 \mathrm{~s}}^{200 \mathrm{w}} d_{\mathrm{SiO} 2}^{200} \mathrm{~nm} @ \mathrm{PPM},(\mathrm{c})$ a $\mathrm{Au}_{15 \mathrm{~s}}^{200 \mathrm{w}} d_{\mathrm{SiO2}}^{200 \mathrm{~nm}} @ \mathrm{PPM}$, and (d) a $t_{\mathrm{Au}}^{100 \mathrm{~nm}} @ \mathrm{Au}_{15 \mathrm{~s}}^{200} \mathrm{~W}_{\mathrm{SiO} 2}^{200 \mathrm{~nm}} @ \mathrm{PPM}$ and close-up images (scale bar: $200 \mathrm{~nm}$ ) of their corresponding surface morphologies.

demonstrate the synergistic effect of the multiple scattering of light in the PC structure and the LSPR caused by AuNC arrays as the presence of "hotspots" by tailoring the nanogaps between adjacent AuNCs of the 3rd-tier of the PPMs. An SERS enhancement up to a factor of $10^{8}$ is experimentally achieved. Additionally, this multilevel hybrid structure has potential in photocatalysis and sensing according to its dual tunability in Bragg mode and plasmonic mode. ${ }^{28,29}$

\section{RESULTS AND DISCUSSION}

2.1. Fabrication of Hierarchical Plasmonic-Photonic Microspheres. Figure 1 shows the process for fabricating $\mathrm{PPMs}^{30}$ serving as SERS-active substrates. In this process, we combine droplet microfluidics with metal film deposition and thermal annealing. Note that the annealing process in this work is different from our previous work ${ }^{28}$ where an inert gas of nitrogen $\left(\mathrm{N}_{2}\right)$ was applied for formation of the AuNC arrays, avoiding surface cross contamination and ensuring the chemical properties. It is of great significance for subsequent molecule immobilization with respect to bioassays. Briefly, photonic crystal microsphere (PMs) were obtained using a microfluidic droplet generator (Figure 1a,b). These PMs possess PSB properties, resulting from the periodic $\mathrm{SiO}_{2} \mathrm{NP}$ arrangements that offer the required periodically modulated permittivity. ${ }^{31} \mathrm{By}$ tailoring the $\mathrm{SiO}_{2} \mathrm{NP}$ diameter $\left(d_{\mathrm{SiO} 2}\right)$, the PSB properties are precisely controlled. Depositing a thin layer of a Au film onto the as-prepared PMs (Figure 1c) and annealing them in $\mathrm{N}_{2}$ at 800 ${ }^{\circ} \mathrm{C}$ for $1 \mathrm{~h}$ results in PPMs consisting of both the PC structures and well-spaced AuNC arrays (Figure 1d). The surface morphology of the AuNC arrays and the AuNC diameters strongly depend on the thickness of the deposited Au films. The formation of AuNCs is attributed to the dewetting of the Au film deposited onto the surface of $\mathrm{SiO}_{2} \mathrm{NP}$ arrangements. ${ }^{32}$ Both the AuNC diameter $\left(d_{\mathrm{Au}}\right)$ and their edge-to-edge distance $\left(p_{\mathrm{Au}}\right)$ increase with increasing $d_{\mathrm{SiO} 2}$ (Figure S1) where PPMs feature one large AuNC on top and multiple small AuNCs along the rim of each $\mathrm{SiO}_{2}$ NP. Subsequent deposition of a second continuous $\mathrm{Au}$ film on these as-prepared PPMs leads to a narrowing of the $p_{\text {Au }}$ (Figure 1e). This can result in a plasmon coupling effect with near-field EM enhancement, thereby creating high density hotspots. ${ }^{33}$

Figure 2 shows top-view high-resolution scanning electron microscopy (HR-SEM) images of surface morphology of the fabricated PM, PPM, and Au-coated PPM. The PPMs show both well-ordered hexagonal $\mathrm{SiO}_{2} \mathrm{NP}$ nanopatterns (indicated by the red dashed hexagons) and AuNC arrays either in an irregular pattern with well-distributed small AuNCs (Figure 2b) or in a hexagonal pattern with one large AuNC on the top of each $\mathrm{SiO}_{2} \mathrm{NP}$ and multiple small ones along the rim of the $\mathrm{SiO}_{2} \mathrm{NP}$ (Figure 2c). In this work, the nomenclatures of $d_{\mathrm{SiO} 2}^{x} \mathrm{~nm} @ \mathrm{PM}$, $\mathrm{Au}_{n \mathrm{~s}}^{m} \mathrm{~W} d_{\mathrm{SiO} 2}^{x} \mathrm{~nm} @ \mathrm{PPM}$, and $t_{\mathrm{Au}}^{y \mathrm{~nm}} @ \mathrm{Au}_{n \mathrm{~s}}^{m} \mathrm{~W} d_{\mathrm{SiO} 2}^{x} \mathrm{~nm} @ \mathrm{PPM}$ are used to clearly label the parameters of the obtained PMs, PPMs, and Aucoated PPMs, respectively. The superscript $m$ and subscript $n$ describe the thin Au film sputtering power and duration, $x$ is the $\mathrm{SiO}_{2} \mathrm{NP}$ diameter, and $y$ is the thickness of the second deposited $\mathrm{Au}$ film. The PM $\left(d_{\mathrm{SiO} 2}^{200} \mathrm{~nm} @ \mathrm{PM}\right.$, Figure 2a) consists of $\mathrm{SiO}_{2} \mathrm{NPs}$ of $200 \mathrm{~nm}$ in diameter, whereas the PPM consists of both $\mathrm{SiO}_{2} \mathrm{NPs}$ of $200 \mathrm{~nm}$ in diameter and AuNCs of $29 \pm 3 \mathrm{~nm}$ $\left(\mathrm{Au}_{5 \mathrm{~s}}^{200 \mathrm{w}} d_{\mathrm{SiO} 2}^{200 \mathrm{~nm}} @ \mathrm{PPM}\right.$, Figure 2b) or $101 \pm 4 \mathrm{~nm}$ $\left(\mathrm{Au}_{15 \mathrm{~s}}^{200 \mathrm{w}} d_{\mathrm{SiO} 2}^{200 \mathrm{~nm}} @ \mathrm{PPM}\right.$, Figure $\left.2 \mathrm{c}\right)$. Coating a second layer of a continuous $\mathrm{Au}$ film of $100 \mathrm{~nm}$ thickness $\left(t_{\mathrm{Au}}=100 \mathrm{~nm}\right)$ onto the as-prepared PPM $\left(\mathrm{Au}_{15 \mathrm{~s}}^{200 \mathrm{w}} d_{\mathrm{SiO} 2}^{200 \mathrm{~nm}} @ \mathrm{PPM}\right.$, Figure $\left.2 \mathrm{c}\right)$ results in the Au-coated PPM $\left(t_{\mathrm{Au}}^{100 \mathrm{~nm}} @ \mathrm{Au}_{15 \mathrm{~s}}^{200} d_{\mathrm{SiO} 2}^{200} \mathrm{~nm} @ P P M\right.$, Figure $\left.2 \mathrm{~d}\right)$. In this manner, the nanogaps among the adjacent AuNCs can be precisely tailored to achieve an EM field enhancement.

2.2. Optical Properties of Hierarchical PlasmonicPhotonic Microspheres. Figure 3 shows optical microscopy (OM) images in bright-field (BF) and dark-field (DF) illumination modes, and their corresponding reflection spectra of the fabricated PMs and PPMs. The detailed optical reflection spectra measurements are described in the Methods section. The PMs demonstrate PSB properties (solid curves in Figure $3 \mathrm{a}, \mathrm{b})$, which can be tailored by varying the $d_{\mathrm{SiO} 2}$. Their Bragg diffraction peaks show a red shift from $444 \mathrm{~nm}\left(d_{\mathrm{SiO} 2}^{200 \mathrm{~nm}} @ \mathrm{PM}\right)$ to $597 \mathrm{~nm}\left(d_{\mathrm{SiO} 2}^{250 \mathrm{~nm}} @ \mathrm{PM}\right)$ and $623 \mathrm{~nm}\left(d_{\mathrm{SiO} 2}^{280 \mathrm{~nm}} @ \mathrm{PM}\right)(\mathrm{BF}$ illumination in Figure $3 \mathrm{a}$ ) with increasing $d_{\mathrm{SiO} 2} \cdot{ }^{34}$ The PSB is 

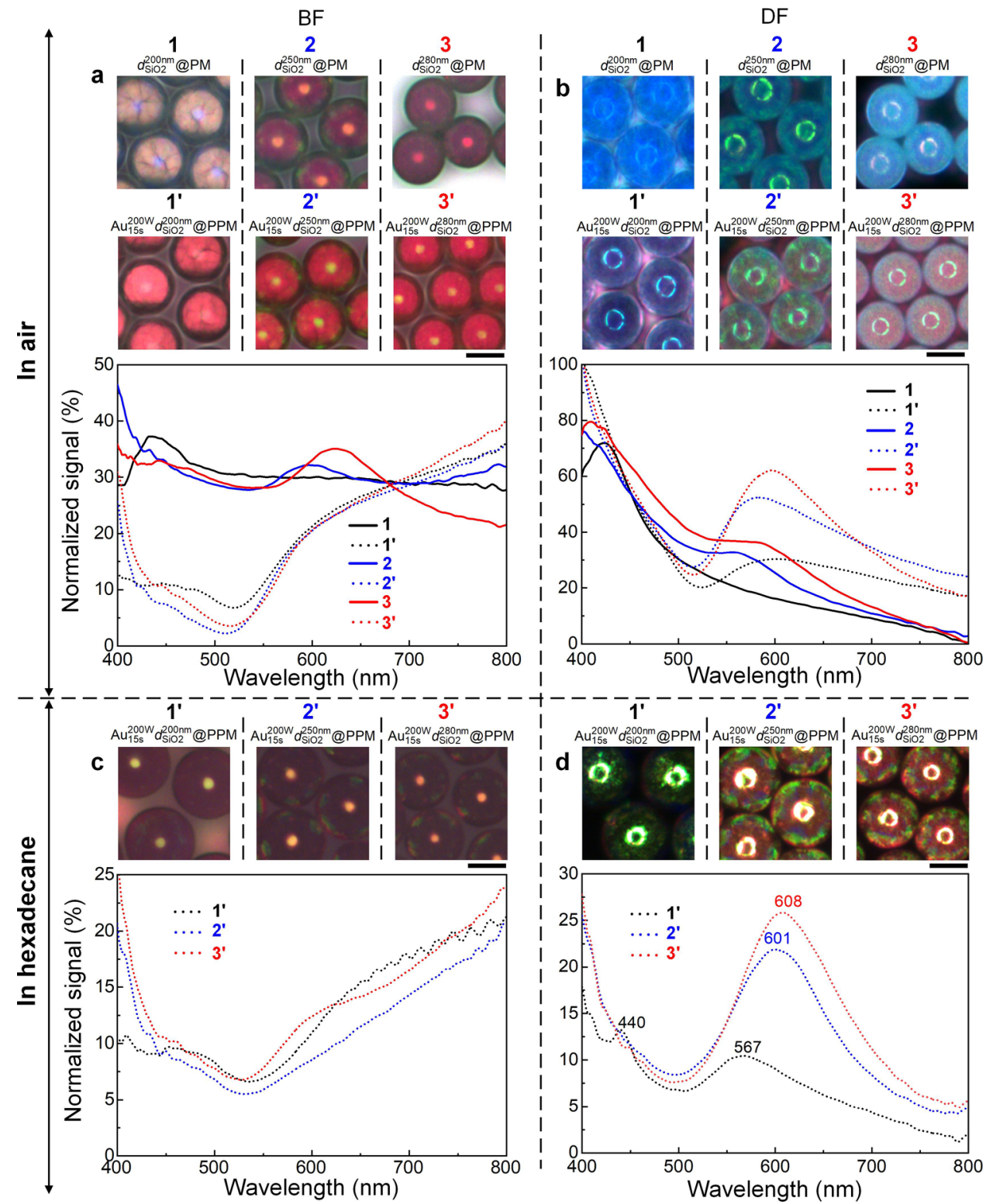

Figure 3. (a, c) BF and (b, d) DF images (scale bar: $10 \mu \mathrm{m}$ ) of PMs (without AuNC arrays) and PPMs (with one large AuNC on each of SiO $\mathrm{NPs}^{\mathrm{N} \text { and }}$ multiple small ones along the rim of $\mathrm{SiO}_{2} \mathrm{NPs}$ ) captured in ambient air (panels (a) and (b)), and hexadecane (panels (c) and (d)) and their corresponding reflection spectra.

clearly visible in these spectra as a local peak that shifts toward longer wavelengths with increasing $d_{\mathrm{SiO} 2}$. Side lobes that would appear in a perfect PC structure with a planar interface are not visible because of the spherical shape of the PC structure and possibly also due to presence of defects and disclinations in the fabricated samples. The PPMs possess both PSB properties (resulting from the PC structures) and SPR properties (resulting from the AuNC arrays). Their reflection spectra (dotted curves in Figure 3a,b) show strong dips at approximately $517 \mathrm{~nm}$ wavelength, resulting from the AuNC array absorption. ${ }^{35}$ Note that a broad scattering peak with a low intensity of the PPMs consisting of $200 \mathrm{~nm} \mathrm{SiO} 2 \mathrm{NPs}\left(\mathrm{Au}_{15 \mathrm{~s}}^{200} \mathrm{w}_{\mathrm{SiO} 2}^{200 \mathrm{~nm}} @ \mathrm{PPMs}\right)$ (black dotted curve in Figure $3 \mathrm{~b}$ ) is observed for which the Bragg diffraction peak (in the blue spectral region/high energy region) is separated from the Au scattering peak (in the red spectral region/low energy region). The scattering peaks from the AuNC arrays on the PPMs consisting of $\mathrm{SiO}_{2} \mathrm{NPs}$ of 250 and $280 \mathrm{~nm}$ in diameter show a red shift and a larger magnitude with increasing the $d_{\mathrm{SiO} 2}$ (blue dotted and red dotted curves in Figure $3 \mathrm{~b}$ ). This is attributed to the overlap of the Bragg diffraction from the PC structures and AuNC array scattering. The different behavior with wavelength of the scattering of the AuNC arrays in DF compared to that in $\mathrm{BF}$ is noteworthy and not yet completely understood. We attribute the difference to the complex 3D structure of the AuNC arrays favoring specular reflection when illuminated from above compared to the more sideway scattering required for the DF signal.

Figure $3 c, d$ shows the $B F$ and DF images and their corresponding reflection spectra of these PPMs measured in hexadecane with a refractive index $(n)$ of 1.4 instead of in ambient air $(n=1.0)$, respectively. We found that these PPMs show similar dips as in air but shift to approximately $530 \mathrm{~nm}$ wavelength (Figure 3c) due to the PPMs with the surrounding medium of hexadecane. Similar to the measurements in ambient air, the scattering peaks of the PPMs in hexadecane (Figure 3d) show a red shift accompanied by a larger magnitude with 


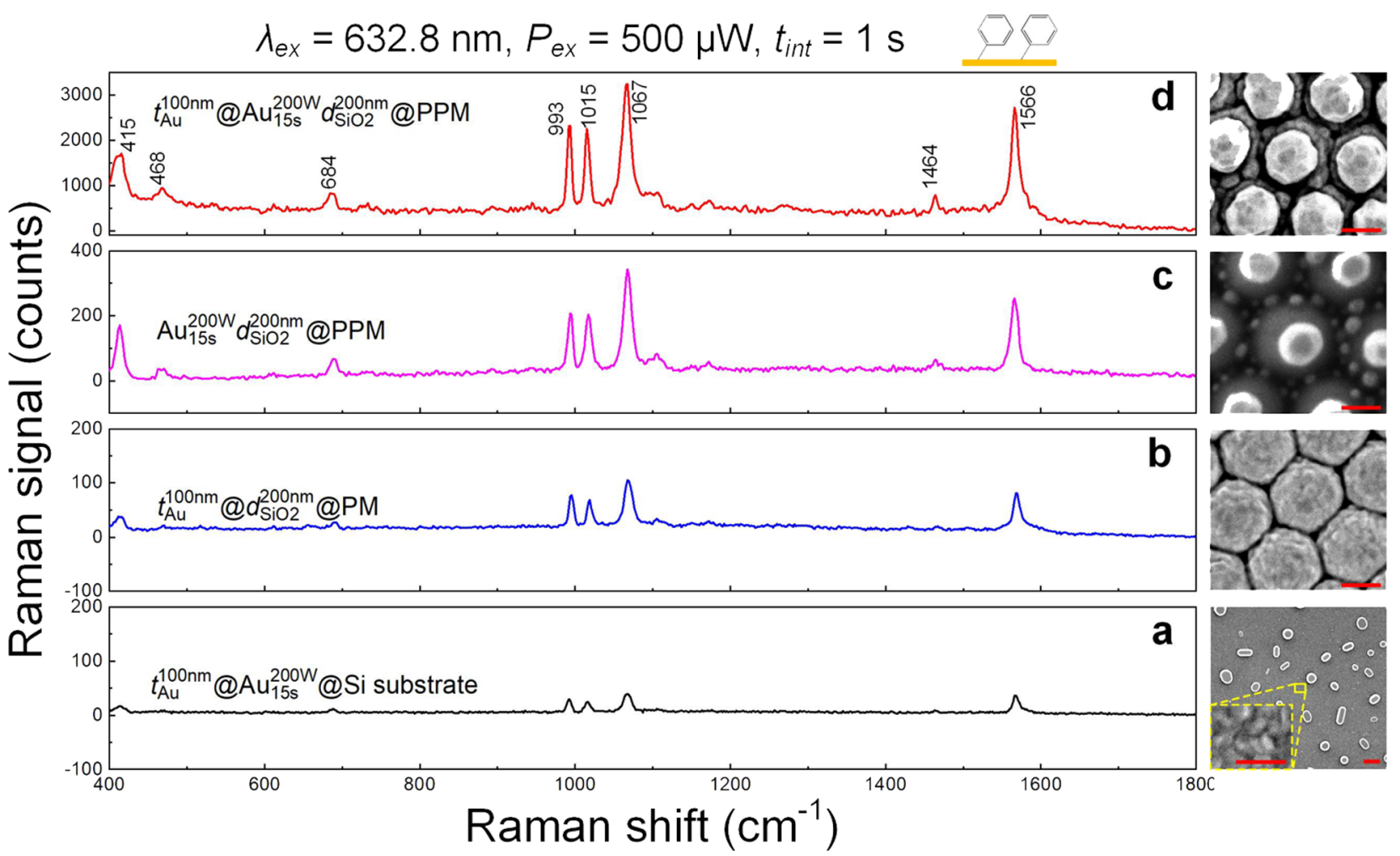

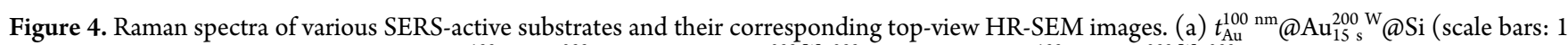
$\mu \mathrm{m}$ and $200 \mathrm{~nm}$ in the close-up image). (b) $t_{\mathrm{Au}}^{100 \mathrm{~nm}} @ d_{\mathrm{SiO} 2}^{200} \mathrm{~nm} @ P M s .(\mathrm{c}) \mathrm{Au}_{15 \mathrm{~s}}^{200 \mathrm{w}} d_{\mathrm{SiO} 2}^{200} \mathrm{~nm} @ P P M s .(\mathrm{d}) t_{\mathrm{Au}}^{100 \mathrm{~nm}} @ \mathrm{Au}_{15 \mathrm{~s}}^{200} d_{\mathrm{SiO} 2}^{200} @ \mathrm{~nm} @ \mathrm{PPM}$. Scale bars represent $100 \mathrm{~nm}$ in panels (b), (c), and (d).

increasing $d_{\mathrm{SiO} 2 \mathrm{NP}}$. This indicates that a red shift of the Bragg diffraction with increasing $d_{\mathrm{SiO} 2 \mathrm{NP}}$ can result in an enhanced $\mathrm{Au}$ scattering (blue and red dotted curves in Figure 3d). The red shift of the scattering peaks is attributed to an increase in $d_{\mathrm{Au}}$. However, when the Bragg diffraction is in the blue spectral region/high energy region (black dotted curve in Figure 3d), Au scattering shows a broad peak with a low intensity in the red spectral region/low energy region, which is consistent with the spectra measured in ambient air. Hereby, it shows high potential to confine light of a specific wavelength to induce a locally amplified EM field incorporated with AuNC arrays for the SERS application, and this hypothesis will be investigated below.

2.3. Hierarchical PPMs as SERS-Active Substrates. Since the fabricated PPMs possess not only multilevel structures ranging from nanometers to micrometers but also integrated optical properties of both PSB and SPR, their SERS performance was explored using a laser emitting at $632.8 \mathrm{~nm}$ for the excitation. Figure 4 shows the Raman spectra of various fabricated SERS-active substrates, including $100 \mathrm{~nm} \mathrm{Au-coated}$ AuNCs randomly distributed on a silicon $(\mathrm{Si})$ wafer $\left(t_{\mathrm{Au}}^{100 \mathrm{~nm}} @\right.$ $\mathrm{Au}_{15}^{200 \mathrm{~s}} @ S i$, Figure 4a), $100 \mathrm{~nm}$ Au-coated PMs ( $t_{\mathrm{Au}}^{100 \mathrm{~nm}} @$ $d_{\mathrm{SiO} 2}^{200} \mathrm{~nm} @ P M s$, Figure 4b), PPMs $\left(\mathrm{Au}_{15 \mathrm{~s}}^{200 \mathrm{w}} d_{\mathrm{SiO} 2}^{200 \mathrm{~nm}} @ P P M s\right.$, Figure $4 \mathrm{c})$, and $100 \mathrm{~nm}$ Au-coated PPMs $\left(t_{\mathrm{Au}}^{100 \mathrm{~nm}} @ \mathrm{Au}_{15 \mathrm{~s}}^{200 \mathrm{w}} d_{\mathrm{SiO} 2}^{200} \mathrm{~nm} @\right.$ PPMs, Figure 4d) and their corresponding top-view HR-SEM images. The Au-coated PPMs $\left(t_{\mathrm{Au}}^{100 \mathrm{~nm}} @ \mathrm{Au}_{15 \mathrm{~s}}^{200 \mathrm{w}} d_{\mathrm{SiO} 2}^{200 \mathrm{~nm}} @ \mathrm{PPMs}\right)$ shows the highest Raman intensity $\left(\mathrm{EF}_{\mathrm{A}}=6.73 \times 10^{7}\right.$ at 1067 $\mathrm{cm}^{-1}$, Table S2) with the dominant Raman active vibrational modes of benzenethiol (BT) molecules. ${ }^{36}$ Note that the slight shifts of vibration frequencies to higher wavenumbers in comparison with the isolated bulk molecules (Figure S2) can be explained by the chemical interaction between the adsorbed molecules and the underneath supporting SERS-active sub- strate. ${ }^{37}$ The calculation of the $\mathrm{EF}_{\mathrm{A}}$ is shown in the Methods section and the Supporting Information where the subscript A indicates that the area occupied by immobilized molecules was equated to the microscope objective spot area. To be more precise, the enhancement factor was also estimated by calculating the BT-immobilized Au surface area, and denoted as $\mathrm{EF}_{\mathrm{Au}}$. The detailed calculation of $\mathrm{EF}_{\mathrm{Au}}$ is shown in the Supporting Information. It is worth noting that the absence of the $909 \mathrm{~cm}^{-1}$ vibration mode in these SERS-active substrates, compared to the normal Raman spectrum of neat BT (Figure S2), indicates the formation of a monolayer of BT molecules on these SERS-active substrates. ${ }^{15}$ Furthermore, a three-dimensional (3D) spatial Raman mapping of $t_{\mathrm{Au}}^{100 \mathrm{~nm}} @ \mathrm{Au}_{15 \mathrm{~s}}^{200 \mathrm{w}} d_{\mathrm{SiO} 2}^{200 \mathrm{~nm}} @$ PPM results in a signal distribution along the $z$ axis by manual focusing with a step of $1 \mu \mathrm{m}$ (Figure S3), suggesting that only the area in focus shows an enhanced signal.

Figure 4 shows that hierarchical microspheres improve the Raman signal, compared to the sample with randomly distributed Au-coated AuNCs on a Si substrate $\left(t_{\mathrm{Au}}^{100 \mathrm{~nm}} @\right.$ $\left.\mathrm{Au}_{15 \mathrm{~s}}^{200 \mathrm{w}} @ \mathrm{Si}\right)$. Both the PPMs $\left(\mathrm{Au}_{15 \mathrm{~s}}^{200 \mathrm{w}} d_{\mathrm{SiO} 2}^{200 \mathrm{~nm}} @ \mathrm{PPMs}\right)$ and the Au-coated PPMs ( $\left.t_{\mathrm{Au}}^{100 \mathrm{~nm}} @ \mathrm{Au}_{15 \mathrm{~s}}^{200 \mathrm{w}} d_{\mathrm{SiO} 2}^{200} \mathrm{~nm} @ P P M s\right)$ show a

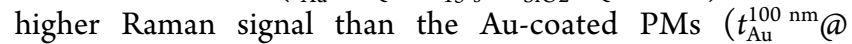
$\left.d_{\mathrm{SiO} 2}^{200} \mathrm{~nm} @ \mathrm{PMs}\right)$. The Raman signal of the Au-coated PMs $\left(t_{\mathrm{Au}}^{100 \mathrm{~nm}} @ d_{\mathrm{SiO} 2}^{200} \mathrm{~nm} @ \mathrm{PMs}\right)$ is caused by the LSPR from high density hotspots and has already been reported in our previous work. $^{11}$ The $100 \mathrm{~nm}$ Au-coated PMs do not offer optimized conditions for producing the amplified EM field caused in these hotspots. The Raman signal of these Au-coated PMs $\left(t_{\mathrm{Au}}^{100 \mathrm{~nm}} @\right.$ $\left.d_{\mathrm{SiO} 2}^{200 \mathrm{~nm}} @ \mathrm{PMs}\right)$ can be maximized by adjusting the nanogap between adjacent metal-coated nanoparticles (Figure S4). When this distance approaches $2 \mathrm{~nm}$, a much stronger EM field can be obtained. ${ }^{15}$ However, in this work, we focus on the 

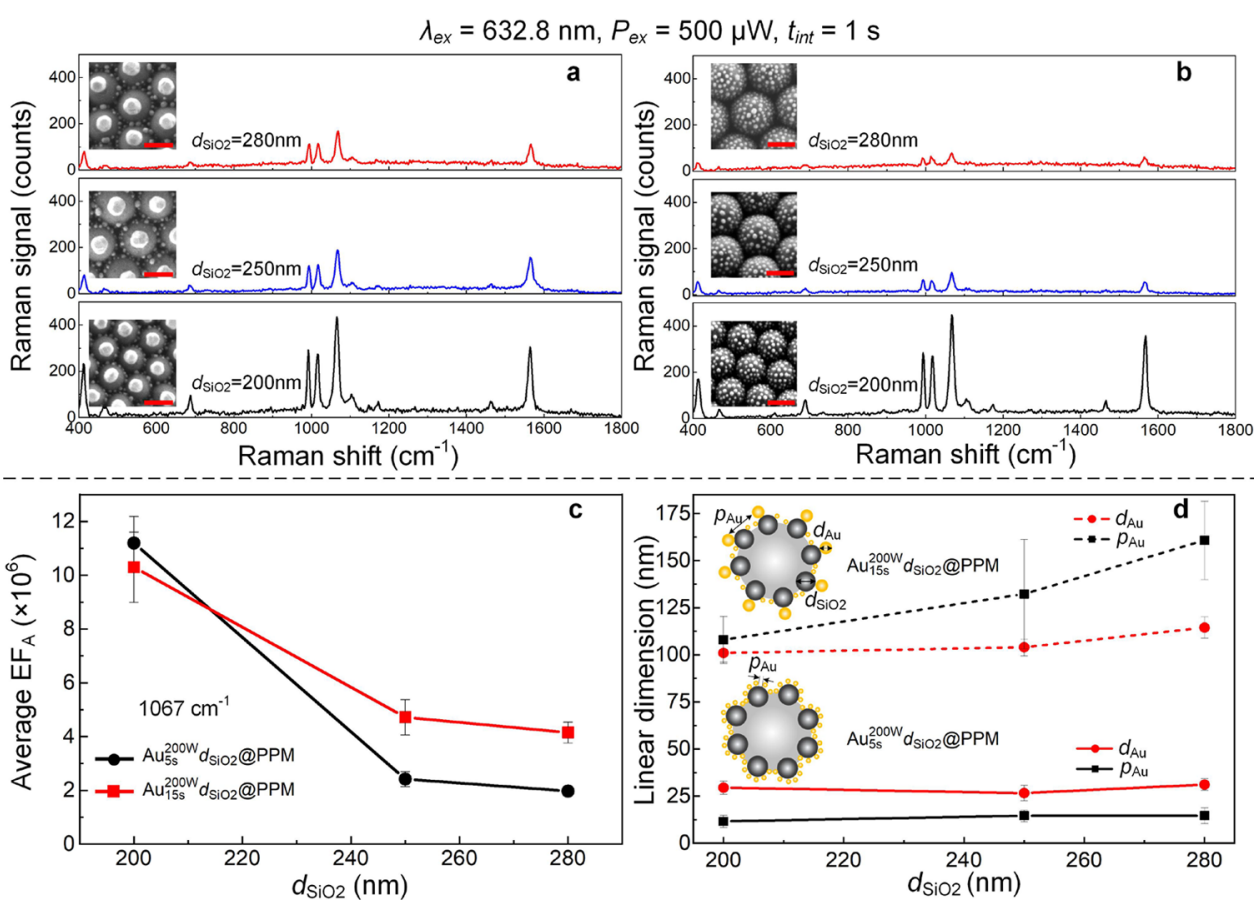

Figure 5. Raman spectra of various PPMs featuring (a) one large AuNC and (b) multiple small AuNCs on top of each $\mathrm{SiO}_{2} \mathrm{NP}$ and their corresponding top-view HR-SEM images (scale bars: $200 \mathrm{~nm}$ ). (c) Average $\mathrm{EF}_{\mathrm{A}}\left(1067 \mathrm{~cm}^{-1}\right)$ of PPMs as a function of the $d_{\mathrm{SiO} 2}$. The error bars were obtained from 10 spectra captured randomly on different microspheres and different locations on each microsphere. (d) $d_{\mathrm{Au}}$ (diameter) and $p_{\mathrm{Au}}$ (gap) of the AuNCs as a function of the $d_{\mathrm{SiO} 2}$, obtained by measuring 20 different diameters and nanogap distances on a single microsphere. Error bars in panels (c) and (d) represent the standard deviation.

Raman enhancement of the PPMs with the purpose to exploit the interplay between the PC structure and the AuNC arrays and the possibility to control the LSPR by further Au coating of the AuNC arrays, which can be precisely controlled in one single

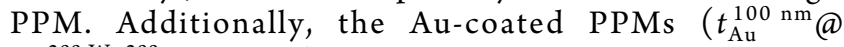
$\left.\mathrm{Au}_{15 \mathrm{~s}}^{200 \mathrm{w}} d_{\mathrm{SiO} 2}^{200 \mathrm{~nm}} @ \mathrm{PPMs}\right)$ show a higher Raman signal than the PPMs without the second $\mathrm{Au}$ layer coating $\left(\mathrm{Au}_{15 \mathrm{~s}}^{200 \mathrm{~W}} d_{\mathrm{SiO} 2}^{200 \mathrm{~nm}} @\right.$ PPMs), indicating the possibility of interplay between the PC structure and the AuNC arrays, which will be further investigated in the following sections. In addition, we should consider the signal contribution from the total Au surface area since Au-coated PPMs have a larger surface area than PPMs without $\mathrm{Au}$ film coating. To quantify this contribution, the $\mathrm{Au}$ surface areas of $\mathrm{Au}_{15 \mathrm{~s}}^{200 \mathrm{w}} d_{\mathrm{SiO} 2}^{200 \mathrm{~nm}} @ P P M s$ and $t_{\mathrm{Au}}^{100 \mathrm{~nm}} @$ $\mathrm{Au}_{15 \mathrm{~s}}^{200 \mathrm{w}} d_{\mathrm{SiO} 2}^{200 \mathrm{~nm}} @ P P M s$ were estimated, obtaining the $\mathrm{EF}_{\mathrm{Au}}$ (Supporting Information). We found that the $\mathrm{EF}_{\mathrm{Au}}(1.80 \times$ $\left.10^{7}\right)$ of $t_{\mathrm{Au}}^{100 \mathrm{~nm}} @ \mathrm{Au}_{15 \mathrm{~s}}^{200 \mathrm{w}} d_{\mathrm{SiO} 2}^{200 \mathrm{~nm}} @ P P M s$ is larger than that of $\mathrm{Au}_{15 \mathrm{~s}}^{200 \mathrm{w}} d_{\mathrm{SiO} 2}^{200} \mathrm{~nm} @ P P M s$ with $\mathrm{EF}_{\mathrm{Au}}=1.20 \times 10^{7}$, which agrees with the variation of $\mathrm{EF}_{\mathrm{A}}$ between these two structures.

2.4. Effect of the PSBs of PPMs on SERS Signals. Here, we investigate the interaction of the PSBs of PPMs with the laser excitation wavelength and its effect on the SERS performance. Figure 5a shows the Raman spectra of the $\mathrm{Au}_{15 \mathrm{~s}}^{200} \mathrm{w}_{\mathrm{SiO} 2}^{x} @ P P M s$ and their corresponding surface morphologies (close-up HRSEM images). The Raman signal increases with decreasing $d_{\mathrm{SiO} 2}$. One possible reason is that the PC structures play a critical role in the Raman enhancement. ${ }^{19}$ PC structures have PSB properties characterized by a Bragg diffraction peak (indicating a spectral region in which the light propagation is forbidden in certain direction) and a slow light effect at the edge of the Bragg diffraction peak with a reduced group velocity. ${ }^{38}$ The sinusoidal standing wave at the blue edge of the Bragg diffraction peak is mainly localized in the low refractive index medium of the PC structures, i.e., in the air voids, whereas at the red edge of the Bragg diffraction peak, the highest amplitude of the standing wave is localized in the high refractive index medium of the PC structures, i.e., in the PC matrix or the matter adsorbed on the PC surface. This implies that adsorbates with a high refractive index confined on the PC structure strongly interact with the light at the red edge region of the Bragg diffraction peak. As discussed above (Figure 3), the Bragg diffraction peaks of the $d_{\mathrm{SiO} 2}^{250} @ \mathrm{~nm} M \mathrm{P}$ and the $d_{\mathrm{SiO} 2}^{280} \mathrm{~nm} @ \mathrm{PMs}$ are located near the laser excitation wavelength, which would suppress the light propagation in such structures. ${ }^{19}$ In contrast, the Bragg diffraction peak of the $d_{\mathrm{SiO} 2}^{200 \mathrm{~nm}} @ \mathrm{PMs}$ is located at $444 \mathrm{~nm}$ (black solid curve in Figure 3a) away from the laser excitation wavelength, so the excitation wavelength cannot be suppressed. Also, the reflection spectrum of the $\mathrm{Au}_{15 \mathrm{~s}}^{200 \mathrm{w}} d_{\mathrm{SiO} 2}^{200 \mathrm{~nm}} @ \mathrm{PPMs}$ under the DF illumination (black dotted curve in Figure $3 \mathrm{~b}$ ) showed AuNCs scattering located at approximately $600 \mathrm{~nm}$ in air. On the other hand, the $\mathrm{Au}_{15 \mathrm{~s}}^{200 \mathrm{w}} d_{\mathrm{SiO} 2}^{250 \mathrm{~nm}} @ \mathrm{PPMs}$ and $\mathrm{Au}_{15 \mathrm{~s}}^{200 \mathrm{~W}} d_{\mathrm{SiO} 2}^{280 \mathrm{~nm}} @ P$ PMs both showed coexistence and mutual enhancement of both Bragg diffraction of PC structures and scattering of AuNC arrays near the excitation wavelength (blue dotted and red dotted curves in Figure 3b,d), which suppresses the light propagation confined in the PPM structures. As a result, we expect that the $\mathrm{Au}_{15 \mathrm{~s}}^{200 \mathrm{w}} d_{\mathrm{SiO} 2}^{200 \mathrm{~nm}} @ \mathrm{PPMs}$ have higher Raman intensity than the $\mathrm{Au}_{15 \mathrm{~s}}^{200 \mathrm{w}} d_{\mathrm{SiO} 2}^{250 \mathrm{~nm} @ P P M s}$ and the $\mathrm{Au}_{15 \mathrm{~s}}^{200 \mathrm{~W}} d_{\mathrm{SiO} 2}^{280} \mathrm{~nm} @ P$ PMs. It should be noted however that the increase in $p_{\mathrm{Au}}$ caused by the increasing $d_{\mathrm{SiO} 2}$ (dashed curves in Figure $5 \mathrm{~d}$ and Figure S1) in this case could possibly lead to a decrease in the Raman signal.

To further study the effect of the PC structures on the Raman signals, PPMs featuring a high density of hotspots with a relatively equivalent $d_{\mathrm{Au}}$ and $p_{\mathrm{Au}}$ are investigated (Figure $5 \mathrm{~b}$ and solid curves in Figure $5 \mathrm{~d}$ ). These PPMs are fabricated by 

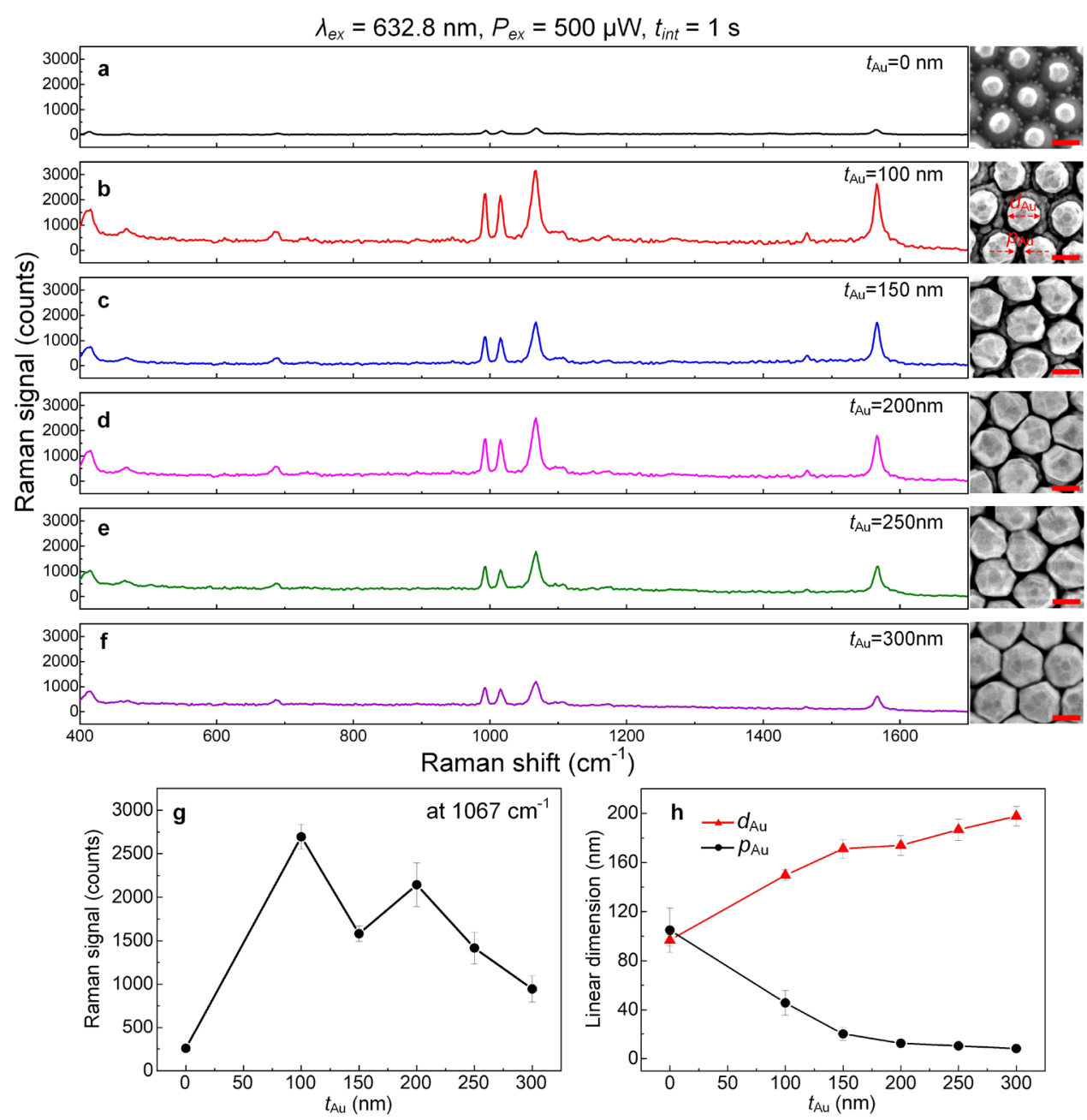

Figure 6. Close-up HR-SEM images (scale bars: $100 \mathrm{~nm}$ ) of $\mathrm{Au}_{15 \mathrm{~s}}^{200} \mathrm{w}_{\mathrm{SiO} 2}^{200 \mathrm{~nm}} @ P P M s$ covered with an Au film of different thicknesses $\left(t_{\mathrm{Au}}\right)(\mathrm{a})$ 0, (b) 100, (c) 150, (d) 200, (e) 250, and (f) $300 \mathrm{~nm}$ and their corresponding Raman spectra. (g) Raman signal at $1067 \mathrm{~cm}^{-1}$ as a function of $t_{\mathrm{Au}}$. The error bars were obtained from 10 spectra captured randomly on different microspheres and different locations on each microsphere. (h) $d_{\mathrm{Au}}$ and $p_{\mathrm{Au}}$ as a function of $t_{\mathrm{Au}}$ obtained by measuring 20 different diameters and nanogap distances on a single microsphere. Error bars in panels $(\mathrm{g})$ and $(\mathrm{h})$ represent the standard deviation.

sputtering a very thin Au film at $200 \mathrm{~W}$ for $5 \mathrm{~s}$ on the as-prepared PMs followed by a dewetting process at $800{ }^{\circ} \mathrm{C}$ for $1 \mathrm{~h}$ in $\mathrm{N}_{2}$ environment. The Raman signals of these PPMs

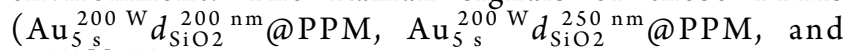
$\mathrm{Au}_{5 \mathrm{~s}}^{200 \mathrm{~W}} d_{\mathrm{SiO} 2}^{280}$ nm @PPM) significantly decrease with increasing $d_{\mathrm{SiO} 2}$ (Figure $5 \mathrm{~b}$ ), which is consistent with the Raman intensity change of the previously used PPMs (Figure 5a,c). The enhancement factors $\left(\mathrm{EF}_{\mathrm{A}}\right)$ of these structures are a function of $d_{\mathrm{SiO} 2}$, as shown in Figure $5 c$, whereby the tendency agrees well with $\mathrm{EF}_{\mathrm{Au}}$ presented in Table S3. We attribute the fact that the $\mathrm{EF}_{\mathrm{Au}}$ of $\mathrm{Au}_{15 \mathrm{~s}}^{200} \mathrm{w}_{\mathrm{SiO}_{2}} @ \mathrm{PPM}$ is larger than the corresponding $\mathrm{EF}_{\mathrm{A}}$ to the fact that the estimated Au surface area is smaller than the used microscope objective spot (calculation details are shown in the Supporting Information). In summary, we found indications that when the PSBs of PPMs are spectrally located near the laser excitation wavelength used in the SERS experiments, the lightmatter interaction is suppressed and the Raman signal is decreased. This agrees with a previous study ${ }^{19}$ and is confirmed by the experimental results.

Additionally, $\mathrm{Au}$-coated monolayers of $\mathrm{SiO}_{2} \mathrm{NPs}$ with different diameters were investigated to further confirm the PSB effect of PPMs on the Raman enhancement (Figure S6). It was found that the Raman signal is not substantially affected by the size of the $\mathrm{SiO}_{2} \mathrm{NPs}$. This can be clearly seen in Figure $\mathrm{S} 6$ where the Raman intensity at $1067 \mathrm{~cm}^{-1}$ as a function of $\mathrm{SiO}_{2} \mathrm{NP}$ diameter is shown. We would like to note that the monolayer of $\mathrm{SiO}_{2} \mathrm{NPs}$ serves as a platform to provide geometrical support for the metallic structures on top but that the exact geometrical dimension in the present regime does not noticeably affect the specific properties of the samples. Furthermore, compared to the PPMs, the Raman signals of the monolayers of close-packed $\mathrm{SiO}_{2} \mathrm{NPs}$ of different diameters were all lower than those of $\mathrm{Au}_{15 \mathrm{~s}}^{200 \mathrm{w}} d_{\mathrm{SiO} 2}^{200} \mathrm{~nm} @ P$ PM but higher than those of $\mathrm{Au}_{15 \mathrm{~s}}^{200 \mathrm{w}} d_{\mathrm{SiO} 2}^{250 \mathrm{~nm}} @$ PPM and $\mathrm{Au}_{15 \mathrm{~s}}^{200 \mathrm{w}} d_{\mathrm{SiO} 2}^{280 \mathrm{~nm}} @ \mathrm{PPM}$. This suggests that the PSBs resulting from the photonic crystals play a role in the localized amplified EM field.

2.5. Synergistic Effect of the Tunable LSPR and PSB on the SERS Signals. The Raman performance of PPMs coated with a layer of the $\mathrm{Au}$ film is investigated for different $\mathrm{Au}$ thicknesses $\left(t_{\mathrm{Au}}\right)$ (Figure 6). The nanogaps between the adjacent nanoparticles can be adjusted by controlling the $t_{\mathrm{Au}}$ which can further enhance the localized EM field by the formation of hotspots. We found a significant change in the obtained Raman signals with an increase in $t_{\mathrm{Au}}$. As $t_{\mathrm{Au}}$ increased from 0 to $300 \mathrm{~nm}$, a change of surface morphology results in the increase of $d_{\mathrm{Au}}$ and decrease of $p_{\mathrm{Au}}$ at the same time (close-up 

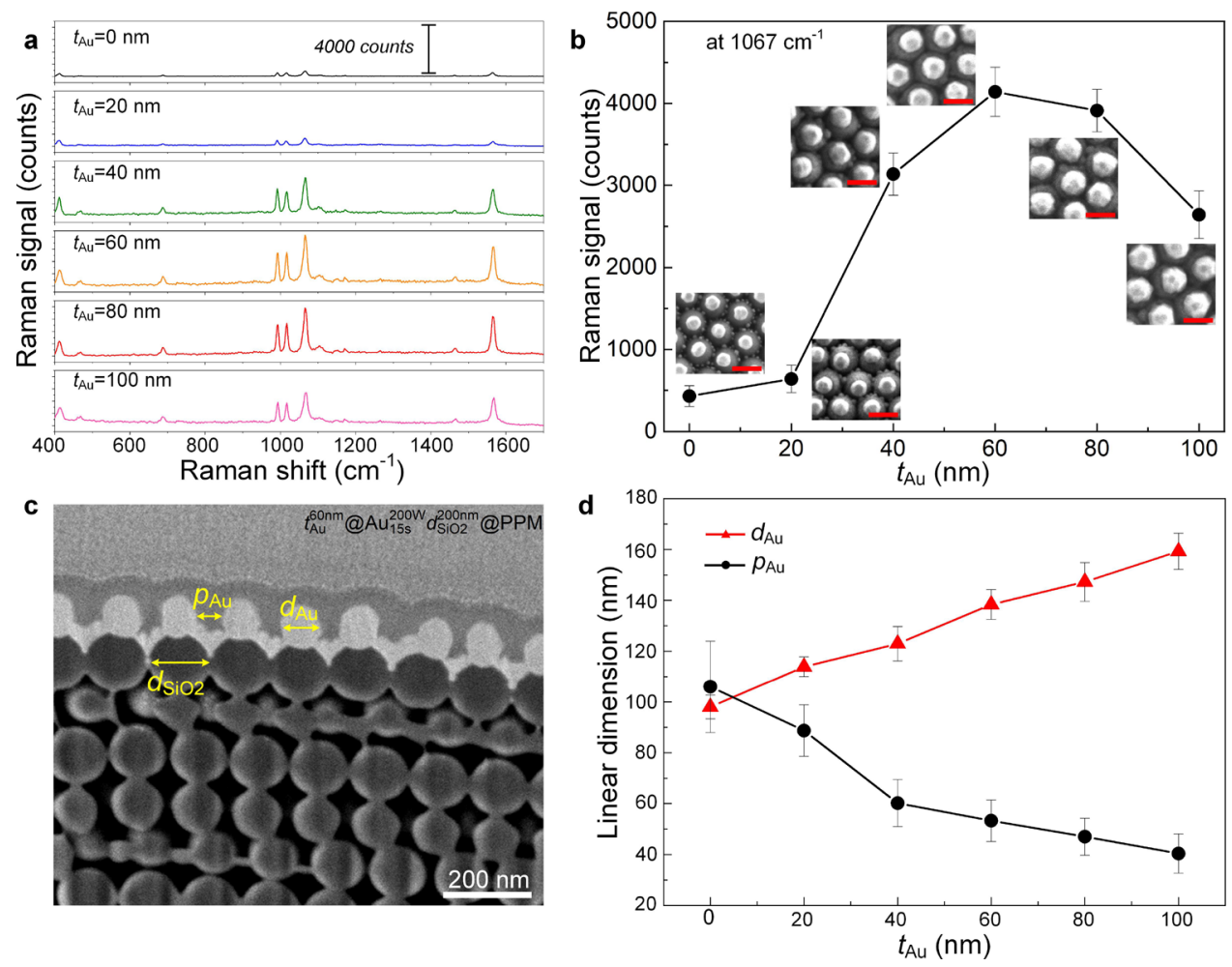

Figure 7. Au-coated PPMs with different $t_{\mathrm{Au}}$ values. (a) Raman spectra and (b) their corresponding Raman intensity at $1067 \mathrm{~cm}^{-1}$ as a function of $t_{\mathrm{Au}}$. The error bars were obtained from 10 spectra captured randomly on different microspheres and different locations per microsphere. Insets are the corresponding HR-SEM top-view images. Scale bar: $200 \mathrm{~nm}$. (c) Cross-sectional HR-SEM images of Au-coated PPMs $\left(t_{\mathrm{Au}}^{60 \mathrm{~nm}} @ \mathrm{Au}_{15 \mathrm{~s}}^{200 \mathrm{w}} d_{\mathrm{SiO} 2}^{200 \mathrm{~nm}} @ P P M\right)$. (d) $d_{\mathrm{Au}}$ and $p_{\mathrm{Au}}$ as a function of $t_{\mathrm{Au}}$ obtained by measuring 20 different diameters and nanogap distances on a single microsphere. Error bars in panels (b) and (d) indicate the standard deviation.

HR-SEM images in Figure $6 a-f$ and $h$ ), which facilitates the formation of hotspots. The Raman signal of Au-coated $t_{\mathrm{Au}}^{y \mathrm{~nm}} \mathrm{Au}_{15 \mathrm{~s}}^{200 \mathrm{w}} d_{\mathrm{SiO} 2}^{200 \mathrm{~nm}} @ P P M s$ with different coating thicknesses $y$ are always higher than that of the uncoated $\mathrm{Au}_{15 \mathrm{~s}}^{200 \mathrm{w}} d_{\mathrm{SiO2} 2}^{200 \mathrm{~nm}} @$

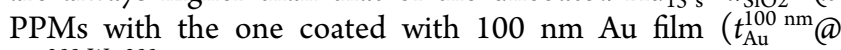
$\left.\mathrm{Au}_{15 \mathrm{~s}}^{200}{ }^{200} d_{\mathrm{SiO} 2} \mathrm{~nm} @ \mathrm{PPM}\right)$ reaching the highest signal. We attribute this to the synergistic effect of the PSB properties with the nearfield plasmon coupling at the hotspots. We hypothesize that the Raman intensity of $t_{\mathrm{Au}}^{150 \mathrm{~nm}} @ \mathrm{Au}_{15 \mathrm{~s}}^{200 \mathrm{w}} d_{\mathrm{SiO} 2}^{200 \mathrm{~nm}} @ \mathrm{PPMs}$ is decreased with respect to the PPMs with $100 \mathrm{~nm}$ Au coating because the increased $\mathrm{Au}$ film started to bury the initial $\mathrm{SiO}_{2} \mathrm{NP}$ lattices, leaving only the AuNC arrays. The light interaction in the PC structures then weakened while another type of PC structure featuring AuNC arrays is formed where the thick Au film behaves like a mirror with high reflection. Figure S7 shows the corresponding optical properties of Au-coated PPMs under the $\mathrm{BF}$ and DF illumination modes, respectively. It is obvious to see that the samples show a dip approximately at $480 \mathrm{~nm}$ caused by a layer of Au film with increasing $t_{\mathrm{Au}}$, especially when $t_{\mathrm{Au}} \geq 200 \mathrm{~nm}$ (Figure S7b). The Raman enhancement of the $t_{\mathrm{Au}}^{150 \mathrm{~nm} @}$ $\mathrm{Au}_{15 \mathrm{~s}}^{200 \mathrm{w}} d_{\mathrm{SiO} 2}^{200 \mathrm{~nm}} @ \mathrm{PPMs}$ then mainly results from the AuNC arrays due to the LSPR occurred in these nanogaps among adjacent $\mathrm{Au}$-coated AuNCs. With further increasing $t_{\mathrm{Au}}$ the Raman intensity increases again, reaching a second maximum for $t_{\mathrm{Au}}^{200 \mathrm{~nm}} @ \mathrm{Au}_{15 \mathrm{~s}}^{200 \mathrm{w}} d_{\mathrm{SiO} 2}^{200 \mathrm{~nm}} @ \mathrm{PPMs}$, and then again decreases with further increasing the $t_{\mathrm{Au}}$. For $t_{\mathrm{Au}} \geq 200 \mathrm{~nm}$, the hexagonal $\mathrm{SiO}_{2} \mathrm{NP}$ nanopatterns are completely covered by the corresponding AuNC arrays so that the Raman enhancement only resulted from the LSPR of the hotspots. The distance of the nanogaps remains relatively stable (HR-SEM images in Figure $6 \mathrm{c}-\mathrm{f}$ and $\mathrm{h})$, but the density of nanogaps decreases as the nanogaps partly joined together (e.g., $\left.t_{\mathrm{Au}}=300 \mathrm{~nm}\right)$, decreasing the surface roughness and resulting in a reduced number of hotspots with decreased Raman signal. Summarizing, we found that the Au-coated PPM with $100 \mathrm{~nm} \mathrm{Au} \mathrm{film} \mathrm{thickness}$ $\left(t_{\mathrm{Au}}^{100 \mathrm{~nm}} @ \mathrm{Au}_{15 \mathrm{~s}}^{200 \mathrm{w}} d_{\mathrm{SiO} 2}^{200 \mathrm{~nm}} @ P P M\right)$ shows the highest Raman intensity. We attribute this to the synergistic effect of the PC structures with the LSPR by hotspot formation. The LSPRinduced amplified EM then dominates the PC structure with increasing $t_{\mathrm{Au}}$ of the Au-coated PPMs.

To more precisely determine the maximal synergistic effect of the PC structure and the LSPR of Au-coated PPMs, the Raman performance of PPMs with a continuous Au film in the range of $0-100 \mathrm{~nm}$ is studied. It was found that the $\mathrm{EF}_{\mathrm{A}}$ reaches a maximum of $10^{8}$ when $t_{\mathrm{Au}}=60 \mathrm{~nm}$ and then decreases again with increasing $t_{\mathrm{Au}}$ (Figure $7 \mathrm{a}, \mathrm{b}$ ). This confirms the hypothesis formulated in the previous section that the amplified EM field raised by the nanogaps between the adjacent AuNCs does not play the dominant role in the explanation of the Raman enhancement. Rather, it is the interplay of field enhancement caused by the PC structures and LSPR. We also found that the $\mathrm{EF}_{\mathrm{A}}$ of Au-coated PPMs is approximately three times larger than the $\mathrm{EF}_{\mathrm{Au}}$ (listed in Table S4), which we attribute to the $\mathrm{Au}$ surface area being larger than the planar surface area on the same projected area (the area of the used microscope objective). However, both the $\mathrm{EF}_{\mathrm{A}}$ and $\mathrm{EF}_{\mathrm{Au}}$ follow the same tendency when varying the Au thickness $t_{\mathrm{Au}}$. Figure $7 \mathrm{c}$ shows the crosssectional view HR-SEM image of $t_{\mathrm{Au}}^{60 \mathrm{~nm}} @ \mathrm{Au}_{15 \mathrm{~s}}^{200 \mathrm{w}} d_{\mathrm{SiO} 2}^{200 \mathrm{~nm}} @ \mathrm{PPM}$. Figure $7 \mathrm{~d}$ shows the $d_{\mathrm{Au}}$ and $p_{\mathrm{Au}}$ varying with $t_{\mathrm{Au}}$.

In addition, the uniformity and reproducibility have also been

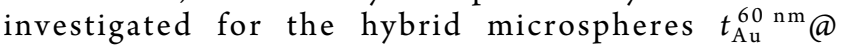
$\mathrm{Au}_{15 \mathrm{~s}}^{200 \mathrm{w}} d_{\mathrm{SiO} 2}^{200 \mathrm{~nm}} @ \mathrm{PPM}$ with the results shown in Figure S8. 

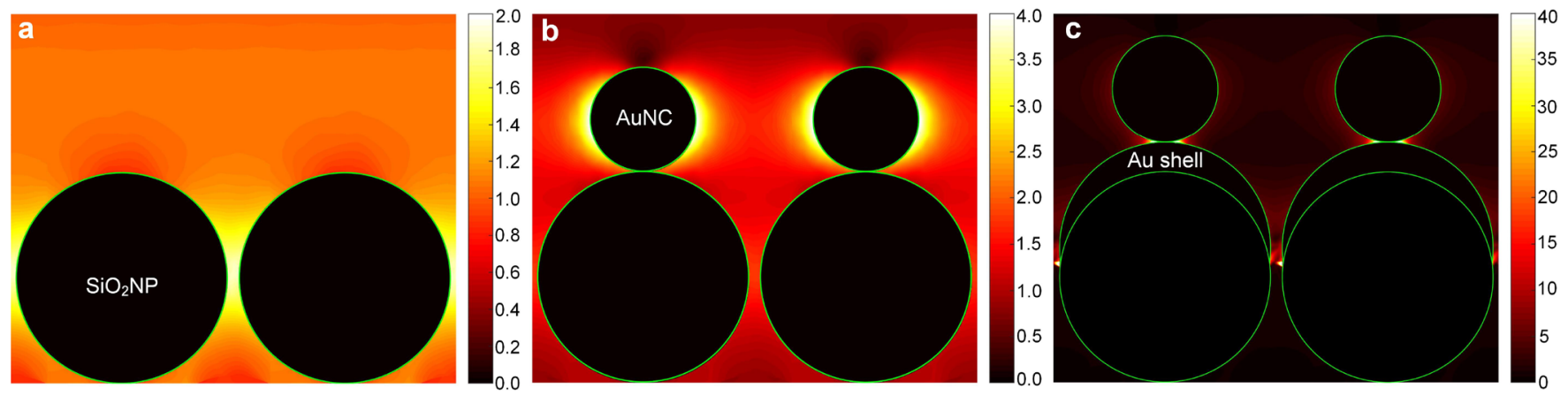

Figure 8. Cross sections of the simulated electric field distribution on different structures. The color bars represent $|E| / \mid E_{0} l$. (a) Pure PM, $d_{\mathrm{SiO} 2}^{200} \mathrm{~nm} @ \mathrm{PM}$. (b) PPM, Au $200 \mathrm{w} d_{\mathrm{SiO} 2}^{200 \mathrm{~nm}} @ P P M$. (c) Au-coated PPM, $t_{\mathrm{Au}} @ \mathrm{Au}_{15 \mathrm{~s}}^{200} \mathrm{w}_{\mathrm{SiO} 2}^{200} \mathrm{~nm} @ P P M$. (panels $(\mathrm{a}-\mathrm{c})$ ) The diameter of constituent $\mathrm{SiO}_{2} \mathrm{NP}$ is $200 \mathrm{~nm}$, and the AuNC is $100 \mathrm{~nm}$ in diameter. Please note that in all figures, the spatial location that we show is on top of an extended PC structure consisting of 20 layers of a hexagonal lattice of $\mathrm{SiO}_{2} \mathrm{NPs}$. The periodicity of $210 \mathrm{~nm}$ is chosen. The green lines indicate the outline of the $\mathrm{SiO}_{2} \mathrm{NPs}_{\mathrm{s}} \mathrm{AuNCs}$, and $\mathrm{Au}$ shells.

SERS spectra were collected from five different microspheres with six different locations per microsphere (Figure S8a). We furthermore investigated the intensity distribution at $1067 \mathrm{~cm}^{-1}$, collected from 20 different microspheres and 20 locations per microsphere of $t_{\mathrm{Au}}^{60 \mathrm{~nm}} @ \mathrm{Au}_{15 \mathrm{~s}}^{200 \mathrm{w}} d_{\mathrm{SiO} 2}^{200 \mathrm{~nm}} @ \mathrm{PPM}$ (Figure S8b) and found a relative standard deviation (RSD) of $12 \%$, demonstrating that the hybrid PPMs can be fabricated with high reproducibility and relative uniformity. Additionally, these hybrid PPMs show potential application in the sensing field, as demonstrated by the small Raman cross-sectional molecule detection using L-methionine (Figure S9).

It remains to be mentioned that an unambiguous assignment of which of the effects causes the observed SERS enhancement is not that obvious. To clear the misunderstanding, we want to indicate these possible effects in a list:

- The spectral alignment of the PSB relative to the excitation wavelength is important due to the aforementioned slow light effect and the localization of the EM field in either the high or the low refractive index region.

- The spectral alignment of the PSB relative to the excitation wavelength also affects the amount of backreflected light and its phase. Considering that the molecules are deposited in a tiny layer above the structure, a fraction of the light that experiences multiple scatterings inside the PC structures is finally back-reflected where it interferes with the illumination. This interference can be either constructive or destructive. This leads to a lowering or enhancement of the local EM field in specific spatial regions. Depending on its alignment with the molecular layer, the SERS signal is enhanced differently.

- Finally, any modification of the PC structure that serves as the backbone for the plasmonic structure changes the geometry of the metallic constituents and with that the exact spectral position of the plasmonic resonances. If the spectral position of the LSPR is in agreement with the wavelength that matters in the SERS process, the signal gets tremendously enhanced.

Even though the disentanglement of the impact of all parameters is too complex and beyond the scope of the present work, we perform full-wave optical simulations of selected structures to discuss the importance of different effects and to verify the experimental findings.

2.6. Full-Wave Numerical Simulations. Figure 8 shows the numerical simulation results of the local electric field of
SERS-active substrates with different relevant geometries. First, we consider a pure photonic microstructure that consists of a hexagonal lattice of $\mathrm{SiO}_{2} \mathrm{NPs}\left(d_{\mathrm{SiO} 2}^{200 \mathrm{~nm}} @ \mathrm{PM}\right.$, Figure 8a). For numerical simulation convenience, the periodicity is chosen to be slightly larger than the $\mathrm{SiO}_{2} \mathrm{NP}$ diameter $(210 \mathrm{~nm})$, which should not affect the conclusion to be drawn. The PC structure is finite and made of 20 layers of $\mathrm{SiO}_{2}$ NPs. It is illuminated at normal incidence with linearly polarized plane wave oscillating at a wavelength of $632.8 \mathrm{~nm}$ according to the experimental condition. The amplitude of the electric field is shown in some selected central cross section, and we concentrate on the spatial region directly above the $\mathrm{PC}$ structure. We furthermore consider a PPM structure $\left(\mathrm{Au}_{15 \mathrm{~s}}^{200 \mathrm{w}} d_{\mathrm{SiO} 2}^{200 \mathrm{~nm}} @ \mathrm{PPM}\right.$, Figure $\left.8 \mathrm{~b}\right)$ where the same PC structure is decorated with AuNCs with diameters of $100 \mathrm{~nm}$. Finally, an Au-coated PPM $\left(t_{\mathrm{Au}} @ \mathrm{Au}_{15}^{200 \mathrm{~s}} d_{\mathrm{SiO} 2}^{200 \mathrm{~nm}} @\right.$ PPM, Figure 8c) is shown. It consists of a crescent type metallic capping, on top of which a metallic AuNC resides. The color scales represent the normalized amplitude of the electric field $|E|$ with respect to the amplitude of the incoming electric field $\left|E_{0}\right|$. Details of the electric field simulations are given in the Methods section.

We found that the maximum enhancement of the local electric field is obtained with the Au-coated PPM (Figure 8c), which is in line with our experimental results (Figure 7). This suggests that the presence of nanogaps between the AuNCs and the $\mathrm{Au}$ shell and in between the shell-coated $\mathrm{SiO}_{2} \mathrm{NPs}$ causes LSPR at the excitation wavelength as used in the SERS experiments, which is mainly responsible for the Raman enhancement. When considering only an isolated AuNC on top of the PC structure, the enhancement is much weaker (Figure $8 b$ ). We do see some field enhancement (amplitude enhanced by a factor of 4). Also, the PC structure alone offers a local enhancement in some spatial regions that is a factor of 2 . This corresponds in good approximation to the value that one would expect at most for a perfect reflector. The illumination is back-reflected and interferes with the incident field in some spatial region, so the total amplitudes can double or can be suppressed. Also, we are operating the PC structure at a wavelength longer than the wavelength of its PSB. The period of the structure is smaller than the wavelength, and the PC structure offers only a zerothdiffraction order. The amplitude of this zeroth-diffraction order gets strongly affected by multiple scattering effects, but it cannot be tremendously enhanced. However, this back-reflected zerothdiffraction order excites the plasmonic structure as well where it gets further enhanced. It is that combined action that explains 
the excellent performance of the suggested structure. So, we exploit not only the plasmonic field due to the excitation with the actual illumination but also the back-reflected field from the PSB structure, adding to the excitation of the plasmonic field and further enhancing the local electric field. As the SERS signal is proportional to the fourth power of the electric field amplitude, this additional field translates to a much higher SERS signal.

In addition, we investigate the effect of the PC structure of Aucoated PPMs on the electric field with varying $d_{\mathrm{SiO} 2}$ (Figure $\mathrm{S} 10 \mathrm{a}-\mathrm{c}$ ). The simulated results agree with our experimentally determined Raman enhancement (Figure S10d), further confirming that the PC structure can improve the Raman signal according to their enhanced light-matter interaction due to the multiple scattering in combination with the plasmonic effect these samples sustain. Herein, the simulated EFs are determined as $|E|^{4} /\left|E_{0}\right|^{4}$ at the spatial location where the electric field is the highest. ${ }^{39}$ Experimental EFs are obtained by the corresponding sample measurements with the maximum Raman signal enhancement. The deviation of the EFs between the simulated and experimentally determined ones is mainly attributed to the imperfection of the PC structures, which can cause differences between the simulated electric field and the real produced electric field. Also, of course, molecules are not just situated in the hotspot but also in other spatial regions. There they contribute as well to the Raman signal. However, it should be noted that the estimated EF according to the simulations is not the spatial average. This could be another point to cause the estimated EF larger than the experimental EF. Nevertheless, the theoretically predicted functional dependency and particularly the conclusion on the most optimal sample agree with the experimental findings.

\section{CONCLUSIONS}

Hierarchical plasmonic-photonic microspheres consisting of the PC structure of close-packed colloids and well-spaced AuNC arrays have been successfully fabricated by using a robust and cost-effective approach with high throughput, designability, and reproducibility. The fabricated PPMs possess both PSB properties resulting from the periodic $\mathrm{SiO}_{2} \mathrm{NP}$ arrangements and LSPR resulting from the Au-coated AuNC arrays. The multilevel structures and optical properties of these fabricated PPMs can be adjusted by tailoring the size of the building blocks and the nanogaps among adjacent AuNCs and thus achieving a synergistic interaction of PSB and LSPR. Moreover, the Raman performance of these PPMs has been evaluated by detection of the chemisorbed monolayer of BT molecules on these fabricated SERS-active units. The obtained enhancement factor is on the order of magnitude of $10^{8}$ for the $1067 \mathrm{~cm}^{-1}$ of the thiol-Au bond vibration. Among these PPMs, the $t_{\mathrm{Au}}^{60 \mathrm{~nm} @}$ $\mathrm{Au}_{15 \mathrm{~s}}^{200 \mathrm{w}} d_{\mathrm{SiO} 2}^{200 \mathrm{~nm}} @$ PPMs show the highest $\mathrm{EF}_{\mathrm{A}}$ of $1.04 \times 10^{8}$ caused by the synergistic interaction of multiple scattering confined in the PC structures and the LSPR of Au-coated AuNC arrays. As a result, microscale SERS-active units can be prepared in a scalable and controllable way, which would widen and improve wearable sensing devices in the future.

\section{METHODS}

4.1. Fabrication of Hierarchical PPMs. PMs consisted of wellordered $\mathrm{SiO}_{2} \mathrm{NPs}$ were fabricated using a droplet-based microfluidic platform (Figure 1a). ${ }^{40}$ The $\mathrm{SiO}_{2} \mathrm{NPs}$ with different diameters $(200 \pm$ $10,250 \pm 10$, and $280 \pm 10 \mathrm{~nm}$ ) were purchased from the Nanjing Rainbow company (Nanjing, China). These PMs (Figure $1 \mathrm{~b}$ ) were then used as templates for manufacturing PPMs via the Au thin film deposition (Figure 1c) and thermal annealing (Figure 1d). The sputtering of Au films was conducted in an ion-beam sputtering system (home-built T'COathy system, MESA+, the Netherlands) at $200 \mathrm{~W}$ and $6.6 \times 10^{-3} \mathrm{mbar}$. The thermal annealing process was conducted in a tube furnace (Nabertherm, Nabertherm $\mathrm{GmbH}$, Germany) in a $\mathrm{N}_{2}$ environment at atmospheric pressure. Sputtering the second Au film onto the as-prepared PMs and PPMs can further modify their structures and optical properties (Figure 1e).

4.2. Reflection Measurements. A monolayer of fabricated microspheres was patterned on a piece of clean silicon wafer. The reflection spectra and corresponding OM images were measured on the pre-patterned samples in the $\mathrm{BF}$ and DF illumination modes, using a microscope with a $20 \times / 0.4 \mathrm{NA}$ objective integrated with a white light source ( $100 \mathrm{~W}$ tungsten xenon lamp) with a light spot size around 1.1 $\mathrm{mm}$ in diameter and an Ocean Optics HR4000 visible fiber optic spectrometer. The reflected light was collected by the same objective and passed through a multimode fiber (QP450-1-XSR, Ocean Optics) to the spectrometer with an integrated detector (HR4000, Ocean Optics). A clean silicon wafer without any patterns was used as $100 \%$ reflection in $\mathrm{BF}$ mode. The observed intensities of samples from the microscope are normalized to the intensity as observed with a clean piece of silicon wafer without any patterns. The used microscope uses a higher illumination intensity in the DF mode than that in the BF mode. The fabricated samples were measured both in ambient air and hexadecane.

4.3. SEM Characterization. A HR-SEM (GeminiSEM 500, Carl Zeiss Microscopy $\mathrm{GmbH}$ ) was used to characterize the surface topology of the fabricated hierarchical microspheres at acceleration voltages ranging from 0.7 to $2 \mathrm{kV}$ with different detectors: secondary electron detector (SE2) or in-lens detector.

4.4. SERS Measurements. Prior to the measurements of Raman spectra, all prepared substrates were immersed into the BT solution (10 $\mathrm{mM}$ ) in ethanol overnight followed by gently rinsing with dehydrated ethanol and passively drying at room temperature. A confocal Raman microscope (Alpha300R, Witech $\mathrm{GmbH}$ ) consisted of a TE-cooled charge-coupled device (CCD) camera (DU970P-BV, Andor Technology, Belfast, Northern Ireland) at $-60^{\circ} \mathrm{C}$ and a $\mathrm{He}-\mathrm{Ne}$ laser $\left(P_{\max }=24\right.$ $\mathrm{mW}$ and $\left.\lambda_{\mathrm{ex}}=632.8 \mathrm{~nm}\right)$, and a UHTS300 spectrometer $(\mathrm{f} / 4300 \mathrm{~mm}$ $\mathrm{FL}$, and grating of 600 lines $\mathrm{mm}^{-1}$ ) was used to collect the Raman spectra. All measurements were performed by using a $100 \times / 0.9 \mathrm{NA}$ microscope objective and a $\mathrm{He}-\mathrm{Ne}$ excitation laser of $632.8 \mathrm{~nm}$ wavelength. Raman spectra of chemisorbed BT on the fabricated SERSactive substrates were measured in ambient air with an excitation laser power of $500 \mu \mathrm{W}$ (measured at the entrance of the microscope objective), an integration time ( $\left.t_{\text {int }}\right)$ of $1 \mathrm{~s}$, and 10 times accumulation. Whereas, the Raman mapping measurements were conducted with an excitation laser power of $500 \mu \mathrm{W}$, an integration time of $1 \mathrm{~s}$, and onetime accumulation. The collected Raman signal was presented in CCD counts. Spatial imaging was performed over $10 \times 10 \mu \mathrm{m}^{2}$ area with 40 lines and 50 measurements per line, yielding a total of 2000 measurements per scanned image. Particularly, as the SERS-active unit was in 3D, the spatial imaging along the $z$ axis was collected in every step of $1 \mu \mathrm{m}$ by manual focusing.

4.5. Calculation of EFs. EFs were calculated by using the equation $\mathrm{EF}=\left(I_{\text {SERS }} / I_{\text {Bulk }}\right) \times\left(N_{\text {Bulk }} / N_{\text {SERS }}\right.$, where $I_{\text {SERS }}$ and $I_{\text {Bulk }}$ are the intensities of the same band of the Raman signal from the analytical molecules on a SERS-active unit and on the pure bulk analyte, respectively, and $N_{\text {SERS }}$ and $N_{\text {Bulk }}$ are the number of the analytical molecules probed on the SERS-active unit and on the pure bulk analyte, respectively. The number of chemisorbed $\mathrm{BT}$ molecules probed on the SERS-active unit is estimated by $N_{\mathrm{SERS}}=A_{\mathrm{s}} D_{\mathrm{BT}}$. $D_{\mathrm{BT}}$ is the surface density of BT molecules chemisorbed on an $\mathrm{Au}(111)$ surface $^{41}$ and equals $3.3 \times 10^{18}$ molecules $\cdot \mathrm{m}^{-2}$. $A_{\mathrm{s}}$ is the surface area of molecules immobilized. Here, either the area of the used microscope objective spot $(1 \mu \mathrm{m}$ in diameter) or the Au surface area of each structure was used to estimate $A_{\mathrm{s}}$ and thus $\mathrm{EF}_{\mathrm{A}}$ and $\mathrm{EF}_{\mathrm{Au}}$ can be obtained. More details on $N_{\text {SERS }}$ is described in the Supporting Information. The number of bulk BT molecules in the confocal volume $\left(V_{\mathrm{CV}}\right)$ of the conventional Raman measurements is estimated by $N_{\text {Bulk }}=$ $N_{\mathrm{A}} M_{\mathrm{BT}}{ }^{-1} \rho_{\mathrm{BT}} V_{\mathrm{cv}} \approx 3.56 \times 10^{10}$ molecules, where $N_{\mathrm{A}}=6.02 \times 10^{23}$ 
molecules mol ${ }^{-1}, M_{\mathrm{BT}}=110.18 \mathrm{~g} \mathrm{~mol}^{-1}$ is the BT molecular weight, $\rho_{\mathrm{BT}}$ is the molecule density, and $V_{\mathrm{CV}} \approx 6.03 \times 10^{-18} \mathrm{~m}^{3}$ is the experimentally determined confocal volume of the measurement system. The collection volume is dependent on the excitation diameter and the effective probe depth of the laser excitation. The effective probe depth $\left(h_{\mathrm{obj}}\right)$ was obtained by adjusting the substrate stage out of the laser focus plane till the disappearance of the peak intensity with a step of $1 \mu \mathrm{m}$ and recording the characteristic silicon peak value at $934 \mathrm{~cm}^{-1}$ by using a laser excitation wavelength of $632.8 \mathrm{~nm}$ and a $100 \times, 0.9$ NA microscope objective. Then, an $h_{\mathrm{obj}}$ value of $7.68 \mu \mathrm{m}$ was obtained in this experiment.

In this work, the Raman band at $1067 \mathrm{~cm}^{-1}$ was chosen to estimate the EFs. The integrated intensity of the conventional Raman spectrum from a neat BT solution in a seal glass vial was approximately 0.55 counts $\left(\lambda_{\mathrm{ex}}=632.8 \mathrm{~nm}, P_{\mathrm{ex}}=500 \mu \mathrm{W}, 10\right.$ times accumulation, and $t_{\text {int }}=$ $1 \mathrm{~s})$. Details of the estimation of the Raman intensity of the neat BT solution are given in the Supporting Information. The average integrated intensity of the SERS measurement was 4142 counts from the SERS-active unit of $t_{\mathrm{Au}}^{60 \mathrm{~nm}} @ \mathrm{Au}_{15 \mathrm{~s}}^{200 \mathrm{w}} d_{\mathrm{SiO} 2}^{200 \mathrm{~nm}} @ \mathrm{PPM}$, and the average $\mathrm{EF}_{\mathrm{A}}$ was approximately $1.04 \times 10^{8}$.

4.6. Details of Full-Wave Numerical Simulations. The electric fields of different structures were simulated using CST Microwave Studio (Computer Simulation Technology: Microwave Studio, Darmstadt, Germany, 2018). The PM is represented by a hexagonal array of $\mathrm{SiO}_{2} \mathrm{NPs}$ with 20 layers (the structure used in Figure 8a). Then, a hexagonally well-spaced AuNC pattern anchoring on the top of the pure photonic structure was used to represent PPM (the structure used in Figure $8 \mathrm{~b}$ ). Also, the Au-coated PPM is modeled by introducing the other layer of the thin Au film before anchoring a hexagonal AuNC pattern on the pure photonic structure, as shown in Figure $8 \mathrm{c}$. The maximum thickness of the Au shell is $d_{\mathrm{SiO} 2 \mathrm{NP}} / 8$. All of the structures used for investigation of the electric field were illuminated by a normal incident plane wave, and the resulting electric field distribution were collected near the interface between the plasmonic nanoparticle arrays and the PC structures, where the largest values of the electric field are expected. All absolute values of the collected electric field were normalized with the ones of a vacuum-filed simulation, i.e., the magnitude of the incident plane wave. The simulated EF can be approximately expressed by $\left(|E| /\left|E_{0}\right|\right)^{4}$ where $|E|$ is the localized electric field and $\left|E_{0}\right|$ is an incident plane wave magnitude used for normalization. In this work, the maximum electric field value $\left|E_{\max }\right|$ is used for $|E|$, and thus the resulting $E F$ is the maximum evaluation for the respective simulation. We also simulated the electric fields of $\mathrm{Au}$-coated PPMs with varying $d_{\mathrm{SiO}_{2}}$ to investigate the effect of the PC structure on the Raman performance, as presented in Figure S9.

\section{ASSOCIATED CONTENT}

\section{SI Supporting Information}

The Supporting Information is available free of charge at https://pubs.acs.org/doi/10.1021/acsami.0c05596.

EF calculation, neat BT Raman spectra, spatial Raman mapping Raman spectra of Au-coated PMs with varying Au film thickness, Raman spectra and optical microscopy and HR-SEM images of Au-coated monolayer of $\mathrm{SiO}_{2} \mathrm{NPs}$, reflection spectra of Au-coated PPMs, uniformity and reproducibility of PPMs, and Raman spectra of L-methionine and simulated electric field distribution of Au-coated PPMs with varying $d_{\mathrm{SiO} 2}$ (PDF)

\section{AUTHOR INFORMATION}

\section{Corresponding Authors}

Juan Wang - National Centre for International Research on Green Optoelectronics \& South China Academy of Advanced Optoelectronics, South China Normal University, 510006 Guangzhou, China; BIOS Lab-on-a-Chip Group, MESA+ Institute for Nanotechnology, Technical Medical Centre \& Max Planck Center for Complex Fluid Dynamics, University of
Twente, 7522 NB Enschede, the Netherlands; (1) orcid.org/ 0000-0002-7334-1733; Email: juan.wang@uwetnte.nl

Lingling Shui - National Centre for International Research on Green Optoelectronics \& South China Academy of Advanced Optoelectronics, South China Normal University, 510006 Guangzhou, China; ○ orcid.org/0000-0001-8517-1535; Email: shuill@m.scnu.edu.cn

\section{Authors}

Hai Le-The - BIOS Lab-on-a-Chip Group, MESA+ Institute for Nanotechnology, Technical Medical Centre \& Max Planck Center for Complex Fluid Dynamics and Physics of Fluids Group, MESA+ Institute for Nanotechnology \& Max Planck Center for Complex Fluid Dynamics, University of Twente, 7522 NB Enschede, the Netherlands; (1) orcid.org/0000-0002-31532937

Theodosios Karamanos - Institute of Theoretical Solid State Physics, Karlsruhe Institute of Technology, 76131 Karlsruhe, Germany

Radius N.S. Suryadharma - Laser Physics and Nonlinear Optics Group, MESA+ Institute for Nanotechnology, University of Twente, 7522 NB Enschede, the Netherlands

Albert van den Berg - BIOS Lab-on-a-Chip Group, MESA+ Institute for Nanotechnology, Technical Medical Centre \& Max Planck Center for Complex Fluid Dynamics, University of Twente, 7522 NB Enschede, the Netherlands

Pepijn W. H. Pinkse - Complex Photonic Systems Group, MESA + Institute for Nanotechnology, University of Twente, 7522 NB Enschede, the Netherlands

Carsten Rockstuhl - Institute of Theoretical Solid State Physics and Institute of Nanotechnology, Karlsruhe Institute of Technology, 76131 Karlsruhe, Germany

Jan C. T. Eijkel - BIOS Lab-on-a-Chip Group, MESA+ Institute for Nanotechnology, Technical Medical Centre \& Max Planck Center for Complex Fluid Dynamics, University of Twente, 7522 NB Enschede, the Netherlands

Loes I. Segerink - BIOS Lab-on-a-Chip Group, MESA+Institute for Nanotechnology, Technical Medical Centre \& Max Planck Center for Complex Fluid Dynamics, University of Twente, 7522 NB Enschede, the Netherlands

Complete contact information is available at: https://pubs.acs.org/10.1021/acsami.0c05596

\section{Author Contributions}

J.W., L.S., J.C.T.E., and L.I.S. designed research. J.W. performed research. H.L.-T. contributed to the Au sputtering. J.W. and P.W.H.P. performed the optical analysis and interpretation of the optical data. T.K., C.R., and R.N.S.S. performed the simulation. All authors contributed to writing the paper.

\section{Notes}

The authors declare no competing financial interest.

\section{ACKNOWLEDGMENTS}

We appreciate the financial support from the National Key Research \& Development Program of China (2016YFB0401502); Special Fund Project of Science and Technology Application in Guangdong (2017B020240002); Science and Technology Program of Guangzhou (no. 2019050001); Pioneers in Healthcare voucher (project Ischemia on chip) of the University of Twente, MST and ZGT in the Netherlands; the Oversea study of Guangzhou Elite Project support in China; and NWA Startup Quantum 
NanoKeys grant (40017607); and the Alexander von Humboldt Foundation and the German Science Foundation (Cluster 3D Matter Made to Order (EXC-2082/1-390761711)).

\section{REFERENCES}

(1) Kneipp, K.; Wang, Y.; Kneipp, H.; Perelman, L. T.; Itzkan, I.; Dasari, R. R.; Feld, M. S. Single Molecule Detection Using SurfaceEnhanced Raman Scattering (SERS). Phys. Rev. Lett. 1997, 78, 16671670.

(2) Nie, S.; Emory, S. R. Probing Single Molecules and Single Nanoparticles by Surface-Enhanced Raman Scattering. Science 1997, 275, 1102-1106.

(3) Campion, A.; Kambhampati, P. Surface-Enhanced Raman Scattering. Chem. Soc. Rev. 1998, 27, 241-250.

(4) Fan, Z.; Kanchanapally, R.; Ray, P. C. Hybrid Graphene Oxide Based Ultrasensitive SERS Probe for Label-Free Biosensing. J. Phys. Chem. Lett. 2013, 4, 3813-3818.

(5) Yang, L.; Gao, M. X.; Zou, H. Y.; Li, Y. F.; Huang, C. Z. Plasmonic $\mathrm{Cu}_{2-\mathrm{x}} \mathrm{S}_{\mathrm{y}} \mathrm{Se}_{1-\mathrm{y}}$ Nanoparticles Catalyzed Click Chemistry Reaction for SERS Immunoassay of Cancer Biomarker. Anal. Chem. 2018, 90, $11728-11733$.

(6) Park, W.-H.; Kim, Z. H. Charge Transfer Enhancement in the SERS of a Single Molecule. Nano Lett. 2010, 10, 4040-4048.

(7) Thomas, M.; Mühlig, S.; Deckert-Gaudig, T.; Rockstuhl, C.; Deckert, V.; Marquetand, P. Distinguishing Chemical and Electromagnetic Enhancement in Surface-Enhanced Raman Spectra: The Case of Para-Nitrothiophenol. J. Raman Spectrosc. 2013, 44, 1497-1505.

(8) Siegfried, T.; Ekinci, Y.; Martin, O. J. F.; Sigg, H. Gap Plasmons and Near-Field Enhancement in Closely Packed Sub-10 nm Gap Resonators. Nano Lett. 2013, 13, 5449-5453.

(9) Gunnarsson, L.; Bjerneld, E. J.; Xu, H.; Petronis, S.; Kasemo, B.; Käll, M. Interparticle Coupling Effects in Nanofabricated Substrates for Surface-Enhanced Raman Scattering. Appl. Phys. Lett. 2001, 78, 802804.

(10) Mühlig, S.; Cialla, D.; Cunningham, A.; März, A.; Weber, K.; Bürgi, T.; Lederer, F.; Rockstuhl, C. Stacked and Tunable Large-Scale Plasmonic Nanoparticle Arrays for Surface-Enhanced Raman Spectroscopy. J. Phys. Chem. C 2014, 118, 10230-10237.

(11) Wang, J.; Jin, M.; Gong, Y.; Li, H.; Wu, S.; Zhang, Z.; Zhou, G.; Shui, L.; Eijkel, J. C. T.; van den Berg, A. Continuous Fabrication of Microcapsules with Controllable Metal Covered Nanoparticle Arrays Using Droplet Microfluidics for Localized Surface Plasmon Resonance. Lab Chip 2017, 17, 1970-1979.

(12) Chang, S.; Eichmann, S. L.; Huang, T.-Y. S.; Yun, W.; Wang, W. Controlled Design and Fabrication of SERS-SEF Multifunctional Nanoparticles for Nanoprobe Applications: Morphology-Dependent SERS Phenomena. J. Phys. Chem. C 2017, 121, 8070-8076.

(13) Yang, S.; Dai, X.; Stogin, B. B.; Wong, T.-S. Ultrasensitive Surface-Enhanced Raman Scattering Detection in Common Fluids. Proc. Natl. Acad. Sci. U. S. A. 2016, 113, 268-273.

(14) Murray, W. A.; Barnes, W. L. Plasmonic Materials. Adv. Mater. 2007, 19, 3771-3782.

(15) Le Thi Ngoc, L.; Jin, M.; Wiedemair, J.; van den Berg, A.; Carlen, E. T. Large Area Metal Nanowire Arrays with Tunable Sub-20 nm Nanogaps. ACS Nano 2013, 7, 5223-5234.

(16) Jradi, S.; Zaarour, L.; Chehadi, Z.; Akil, S.; Plain, J. Femtosecond Direct Laser-Induced Assembly of Monolayer of Gold Nanostructures with Tunable Surface Plasmon Resonance and High Performance Localized Surface Plasmon Resonance and Surface Enhanced Raman Scattering Sensing. Langmuir 2018, 34, 15763-15772.

(17) Yang, G.; Hu, L.; Keiper, T. D.; Xiong, P.; Hallinan, D. T., Jr. Gold Nanoparticle Monolayers with Tunable Optical and Electrical Properties. Langmuir 2016, 32, 4022-4033.

(18) Tan, Y.; Gu, J.; Xu, W.; Chen, Z.; Liu, D.; Liu, Q.; Zhang, D. Reduction of $\mathrm{CuO}$ Butterfly Wing Scales Generates $\mathrm{Cu}$ SERS Substrates for DNA Base Detection. ACS Appl. Mater. Interfaces 2013, 5, 9878-9882.
(19) Qi, D.; Lu, L.; Wang, L.; Zhang, J. Improved SERS Sensitivity on Plasmon-Free $\mathrm{TiO}_{2}$ Photonic Microarray by Enhancing Light-Matter Coupling. J. Am. Chem. Soc. 2014, 136, 9886-9889.

(20) Hwang, H.; Kim, S.-H.; Yang, S.-M. Microfluidic Fabrication of SERS-Active Microspheres for Molecular Detection. Lab Chip 2011, 11, 87-92.

(21) Krauss, T. F.; De La Rue, R. M.; Brandt, S. Two-Dimensional Photonic-Bandgap Structures Operating at near-Infrared Wavelengths. Nature 1996, 383, 699-702.

(22) Stein, A.; Wilson, B. E.; Rudisill, S. G. Design and Functionality of Colloidal-Crystal-Templated Materials-Chemical Applications of Inverse Opals. Chem. Soc. Rev. 2013, 42, 2763-2803.

(23) Pasquale, A. J.; Reinhard, B. M.; Dal Negro, L. Engineering Photonic-Plasmonic Coupling in Metal Nanoparticle Necklaces. ACS Nano 2011, 5, 6578-6585.

(24) Liu, J.-N.; Huang, Q.; Liu, K.-K.; Singamaneni, S.; Cunningham, B. T. Nanoantenna-Microcavity Hybrids with Highly Cooperative Plasmonic-Photonic Coupling. Nano Lett. 2017, 17, 7569-7577.

(25) Fränzl, M.; Moras, S.; Gordan, O. D.; Zahn, D. R. T. Interaction of One-Dimensional Photonic Crystals and Metal Nanoparticle Arrays and Its Application for Surface-Enhanced Raman Spectroscopy. J. Phys. Chem. C 2018, 122, 10153-10158.

(26) Mu, Z.; Gu, H.; Zhang, B.; Zheng, J.; Zhai, Z.; He, X.; Zhao, Y. FDTD Modeling of Photonic Crystal-Incorporated Gold Nanoparticles for Enhancing the Localized Electric Field. J. Mater. Chem. C 2017, 5, 9540-9544.

(27) Hyam, R. S.; Jeon, J.; Chae, S.; Park, Y. T.; Kim, S. J.; Lee, B.; Lee, C.; Choi, D. Plasmonic-Photonic Interference Coupling in Submicrometer Amorphous $\mathrm{TiO}_{2}-\mathrm{Ag}$ Nanoarchitectures. Langmuir 2017, 33, 12398-12403.

(28) Liu, J.; Zhao, H.; Wu, M.; Van der Schueren, B.; Li, Y.; Deparis, O.; Ye, J.; Ozin, G. A.; Hasan, T.; Su, B.-L. Slow Photons for Photocatalysis and Photovoltaics. Adv. Mater. 2017, 29, 1605349.

(29) Zhao, Y.; Zhao, X.; Gu, Z. Photonic Crystals in Bioassays. Adv. Funct. Mater. 2010, 20, 2970-2988.

(30) Wang, J.; Le-The, H.; Shui, L.; Bomer, J. G.; Jin, M.; Zhou, G.; Mulvaney, P.; Pinkse, P. W. H.; van den Berg, A.; Segerink, L. I.; Eijkel, J. C. T. Multilevel Spherical Photonic Crystals with Controllable Structures and Structure-Enhanced Functionalities. Adv. Opt. Mater. 2020, 8, 1902164.

(31) Tarhan, I. İ.; Watson, G. H. Photonic Band Structure of Fcc Colloidal Crystals. Phys. Rev. Lett. 1996, 76, 315.

(32) Meshot, E. R.; Zhao, Z.; Lu, W.; Hart, A. J. Self-Ordering of Small-Diameter Metal Nanoparticles by Dewetting on Hexagonal Mesh Templates. Nanoscale 2014, 6, 10106-10112.

(33) Huang, Z.; Meng, G.; Huang, Q.; Yang, Y.; Zhu, C.; Tang, C. Improved SERS Performance from Au Nanopillar Arrays by Abridging the Pillar Tip Spacing by Ag Sputtering. Adv. Mater. 2010, 22, 41364139.

(34) Zhao, Y.; Xie, Z.; Gu, H.; Zhu, C.; Gu, Z. Bio-Inspired Variable Structural Color Materials. Chem. Soc. Rev. 2012, 41, 3297-3317.

(35) Eustis, S.; El-Sayed, M. A. Why Gold Nanoparticles Are More Precious than Pretty Gold: Noble Metal Surface Plasmon Resonance and Its Enhancement of the Radiative and Nonradiative Properties of Nanocrystals of Different Shapes. Chem. Soc. Rev. 2006, 35, 209-217.

(36) Joo, T. H.; Kim, M. S.; Kim, K. Surface-Enhanced Raman Scattering of Benzenethiol in Silver Sol. J. Raman Spectrosc. 1987, 18, $57-60$.

(37) Li, Z.; Gosztola, D. J.; Sun, C.-J.; Heald, S. M.; Sun, Y. Exceptional Enhancement of Raman Scattering on Silver Chlorobromide Nanocube Photonic Crystals: Chemical and Photonic Contributions. J. Mater. Chem. C 2015, 3, 2455-2461.

(38) Nishimura, S.; Abrams, N.; Lewis, B. A.; Halaoui, L. I.; Mallouk, T. E.; Benkstein, K. D.; van de Lagemaat, J.; Frank, A. J. Standing Wave Enhancement of Red Absorbance and Photocurrent in Dye-Sensitized Titanium Dioxide Photoelectrodes Coupled to Photonic Crystals. J. Am. Chem. Soc. 2003, 125, 6306-6310.

(39) Giannini, V.; Fernández-Domínguez, A. I.; Heck, S. C.; Maier, S. A. Plasmonic Nanoantennas: Fundamentals and Their Use in 
Controlling the Radiative Properties of Nanoemitters. Chem. Rev. 2011, 111, 3888-3912.

(40) Wang, J.; Le-The, H.; Wang, Z.; Li, H.; Jin, M.; van den Berg, A.; Zhou, G.; Segerink, L. I.; Shui, L.; Eijkel, J. C. T. Microfluidics Assisted Fabrication of Three-Tier Hierarchical Microparticles for Constructing Bioinspired Surfaces. ACS Nano 2019, 13, 3638-3648.

(41) Wan, L.-J.; Terashima, M.; Noda, H.; Osawa, M. Molecular Orientation and Ordered Structure of Benzenethiol Adsorbed on Gold(111). J. Phys. Chem. B 2000, 104, 3563-3569. 